\title{
Credit Derivatives Pricing with Stochastic Volatility Models
}

\author{
Carl Chiarella \\ Finance Discipline Group, UTS Business School \\ University of Technology, Sydney \\ P.O. Box 123, Broadway, NSW 2007, Australia \\ carl.chiarella@uts.edu.au \\ Samuel Chege Maina \\ E.ON Energy Trading SE \\ Holzstrasse 6, 40221 Dusseldorf, Germany \\ samuel.chege-maina@eon.com

\begin{abstract}
Christina Nikitopoulos Sklibosios*
Finance Discipline Group, UTS Business School

University of Technology, Sydney

P.O. Box 123, Broadway, NSW 2007, Australia

christina.nikitopoulos@uts.edu.au
\end{abstract}

March 24, 2013

\begin{abstract}
This paper proposes a model for pricing credit derivatives in a defaultable HJM framework. The model features hump-shaped, level dependent, and unspanned stochastic volatility, and accommodates a correlation structure between the stochastic volatility, the default-free interest rates, and the credit spreads. The model is finite-dimensional, and leads (a) to exponentially affine default-free and defaultable bond prices, and (b) to an approximation for pricing credit default swaps and swaptions in terms of defaultable bond prices with varying maturities. A
\end{abstract}

${ }^{*}$ Corresponding author. 
numerical study demonstrates that the model captures stylized various features of credit default swaps and swaptions.

Key Words: Stochastic volatility; Heath-Jarrow-Morton framework; defaultable bond prices; credit spreads; CDS rates.

\section{Introduction}

Following the events of the recent financial crisis, both the regulators and credit risk modellers have attributed special attention to better understand credit risk markets and to further advance the models used to price and hedge the highly risky elusive instruments traded in these markets. Several key characteristics of the interest rate markets which are closely related to the credit risk markets have been identified in the literature. These markets feature unspanned stochastic volatility (Casassus, CollinDufresne and Goldstein (2005) and Li and Zhao (2006)) which is hump-shaped (Reno and Uboldi (2005)). By using a default-free term structure model, Trolle and Schwartz (2009) identified unspanned volatility components and demonstrate that both humpshaped term structure and unspanned stochastic volatility components are necessary to satisfactory match the implied cap skews and the implied volatilities. Furthermore, innovations in interest rates are correlated with innovations in interest rate volatility (Ball and Torous (1999)) as well as to innovations in the credit spreads (D'Souza, AmirAtefi and Racheva-Jotova (2004) and Berndt, Ritchken and Sun (2010)).

Motivated by the prevalent empirical evidence, we propose a generalised defaultable term structure model within the Heath, Jarrow and Morton (1992) (hereafter HJM) framework that accommodates unspanned hump-shaped stochastic volatility. By construction, the model is consistent with the currently observed yield curve and credit spread curve. The connection between the default-free and the defaultable market is established through the credit spread (Schönbucher (1998)), which allows us to accommodate a correlation structure between the interest rates, the credit spreads and the stochastic volatility.

It is well-known that these models are Markovian in the entire yield curve and credit spread curve thus requiring an infinite number of state variables. Consequently, we propose a generalised volatility specification for the default-free and the defaultable term structure that leads to finite dimensional realisation of the state space, along the lines of Björk, Landén and Svensson (2004), Chiarella and Kwon (2000) and Chiarella, 
Nikitopoulos-Sklibosios and Schlögl (2007). The proposed volatility structure allows for level dependency and hump-shaped shocks and it is driven by an exogenous Markov process. In this regard, our model can be considered as an extension of the Berndt et al. (2010) model to accommodate unspanned stochastic volatility. Motivated by the empirical evidence presented in Chan, Karolyi, Longstaff and Sanders (1992), Amin and Morton (1995) and Mercurio and Moraleda (2000), the volatility structure depends on the level of the short rates and the short-term credit spreads. Under these volatility specifications, the model offers tractability and flexibility as it allows (default-free and defaultable) bond prices to be expressed as exponentially affine functions of state variables which are jointly Markovian. Although the model gives rise to a large yet finite number of state variables, their Markovian structure guarantees that the computational cost remains low. We also derive pricing formulas for single-name credit default swap rates (hereafter CDS rates) and swaptions. Based on approximations proposed by Brigo and Morini (2005), CDS rates are expressed in terms of defaultable bond prices with varying maturities. Following the methodology of Rutkowski and Armstrong (2009), swaptions are also priced by using a Black-type formula.

We finally undertake an extensive sensitivity analysis aiming to gauge the impact of the full correlation structure and the stochastic volatility specifications on the distribution of the defaultable bond prices, on the CDS rates and on the swaption prices. The results indicate that the correlation between the interest rate and credit spread impacts the CDS rate and consequently the swaption prices, similar to the results derived by Berndt et al. (2010) but the impact of the stochastic volatility process parameters is more profound.

The model offers a general yet tractable framework to analyse and measure the extent to which volatility can be spanned in credit risk markets. To our knowledge there is no empirical study on this very important feature of credit risk volatility. This paper provides a theoretical framework based on a state space representation that could be adapted to the study and estimation of stochastic volatility in defaultable markets. This task we leave to future research.

The paper is structured as follows. Section 2 presents a defaultable term structure model with unspanned stochastic volatility. Section 3 proposes a volatility term structure that produces finite dimensional realisations and exponentially affine defaultable bond prices. Section 4 considers the pricing of credit default swaps and credit default swaptions. The sensitivity analysis is carried out in Section 5, Technical details are relegated to the appendices. 


\section{A Defaultable Term Structure Model with Stochas- tic Volatility}

We consider the filtered probability space $\left(\Omega, \mathcal{F},\left(\mathcal{F}_{t}\right)_{0 \leq t \leq T}, \mathbb{P}\right)$, where the filtration $\mathcal{F}_{t}=\mathcal{F}_{t}^{W} \vee \mathcal{F}_{t}^{N}, t \geq 0$ satisfies the usual conditions. We represent with $\mathcal{F}_{t}^{W}$ all the default-free background information generated by a standard $\mathbb{P}$-Wiener process $W(t)$, where $\mathbb{P}$ is the real-world probability measure and $\mathcal{F}_{t}^{N}$ captures the default information where the default time $\tau$ is modelled as a stopping time defined by the first jump time of a Cox (doubly stochastic Poisson) process $N(t)$ with intensity $h(t)$.

The price at time $t$ of the zero-coupon default-free and zero-recovery defaultable bond with maturity $T>t$ are denoted as $P(t, T, \omega)$ and $P^{d}(t, T, \omega)$ respectively, where $\omega \in \Omega$ represents the dependence on stochastic factors such as default-free short rates, defaultable short rates and volatility.

Definition 2.1 1. The instantaneous default-free and defaultable forward rate of interest prevailing at time $t \in[0, T]$ for instantaneous borrowing at $T$, are defined respectively as

$$
\begin{aligned}
f(t, T, \omega) & =-\frac{\partial}{\partial T} \ln P(t, T, \omega), \\
f^{d}(t, T, \omega) & =-\frac{\partial}{\partial T} \ln P^{d}(t, T, \omega) .
\end{aligned}
$$

2. The instantaneous default-free and defaultable short rate are then defined respectively as $r(t, \omega)=f(t, t, \omega)$ and $r^{d}(t, \omega)=f^{d}(t, t, \omega)$.

3. The continuously compounded instantaneous forward credit spread is defined as

$$
\lambda(t, T, \omega)=f^{d}(t, T, \omega)-f(t, T, \omega),
$$

thus the instantaneous short-term credit spread is defined as $c(t, \omega)=\lambda(t, t, \omega)$.

To allow for various factors in the economy or in the market to impact the dynamics of the forward rate and credit spread, we propose a multi-dimensional setup embedding a stochastic volatility process $\mathbb{V}(t)$. We assume that the default-free forward rate curve is driven by $n$ sources of uncertainty, while the (apparently larger) defaultable forward rate curve will be driven by $2 n$ sources of uncertainty. The volatility of these forward rate curves, in general, is driven by a total number of $3 n$ sources of uncertainty, subject to the correlation structure between the forward rates and their volatilities 1

\footnotetext{
${ }^{1}$ In the working paper Chiarella, Maina and Nikitopoulos-Sklibosios (2011), we start with a system
} 
Assumption 2.1 The instantaneous default-free forward rate $f(t, T, \omega)$ and the instantaneous forward credit spread $\lambda(t, T, \omega)$ satisfy the stochastic differential equations

$$
\begin{aligned}
& d f(t, T, \omega)=\alpha^{f}(t, T, \omega) d t+\sum_{i=1}^{n} \sigma_{i}^{f}(t, T, \omega) d W_{i}(t), \\
& d \lambda(t, T, \omega)=\alpha^{\lambda}(t, T, \omega) d t+\sum_{i=1}^{n} \rho_{i}^{f \lambda} \sigma_{i}^{\lambda}(t, T, \omega) d W_{i}(t) \\
& +\sum_{i=n+1}^{2 n} \sqrt{1-\left(\rho_{i-n}^{f \lambda}\right)^{2}} \sigma_{i-n}^{\lambda}(t, T, \omega) d W_{i}(t),
\end{aligned}
$$

The stochastic volatility process $\mathbb{V}(t)=\left\{\left(V_{1}(t), \ldots, V_{n}(t)\right), t \in[0, T]\right\}$ satisfies the set of the stochastic differential equations

$$
d V_{i}(t)=\alpha_{i}^{V}\left(t, V_{i}\right) d t+z_{i}^{V_{1}} \sigma_{i 1}^{V}\left(t, V_{i}\right) d W_{i}(t)+z_{i}^{V_{2}} \sigma_{i 2}^{V}\left(t, V_{i}\right) d W_{n+i}(t)+z_{i}^{V_{3}} \sigma_{i 3}^{V}\left(t, V_{i}\right) d W_{2 n+i}(t),
$$

where, for $i=1, \ldots, n, j=1,2,3$ and for $\left(\rho_{i}^{f \lambda}\right)^{2} \neq 1$, the correlation parameters $z_{i}^{V_{j}}$ are given by

$$
\left\{z_{i}^{V_{1}}=\rho_{i}^{f V}, \quad z_{i}^{V_{2}}=\frac{\rho_{i}^{V \lambda}-\rho_{i}^{f \lambda} \rho_{i}^{f V}}{\sqrt{1-\left(\rho_{i}^{f \lambda}\right)^{2}}}, \quad z_{i}^{V_{3}}=\sqrt{\frac{1-\left(\rho_{i}^{f \lambda}\right)^{2}-\left(\rho_{i}^{f V}\right)^{2}-\left(\rho_{i}^{V \lambda}\right)^{2}+2 \rho_{i}^{f \lambda} \rho_{i}^{f V} \rho_{i}^{V \lambda}}{1-\left(\rho_{i}^{f \lambda}\right)^{2}}} .\right.
$$

Then from (2.3), the defaultable forward rate follows the stochastic differential equation

$$
d f^{d}(t, T, \omega)=\left(\alpha^{f}(t, T, \omega)+\alpha^{\lambda}(t, T, \omega)\right) d t+\sum_{i=1}^{2 n}\left(\tilde{\sigma}_{i}^{f}(t, T, \omega)+\tilde{\sigma}_{i}^{\lambda}(t, T, \omega)\right) d W_{i}(t),
$$

where $\tilde{\sigma}_{i}^{f}(t, T, \omega)=\sigma_{i}^{f}(t, T, \omega)$, for $i=1, \ldots, n$ and zero otherwise, while

$$
\tilde{\sigma}_{i}^{\lambda}(t, T, \omega)= \begin{cases}\rho_{i}^{f \lambda} \sigma_{i}^{\lambda}(t, T, \omega), & \text { for } i=1, \ldots, n \\ \sqrt{1-\left(\rho_{i-n}^{f \lambda}\right)^{2}} \sigma_{i-n}^{\lambda}(t, T, \omega), & \text { for } i=n+1, \ldots, 2 n .\end{cases}
$$

The system (2.4), (2.9) and (2.7) entails the element of unspanned stochastic volatility in a traditional defaultable HJM framework. The defaultable forward curve is driven by $2 n$ factors, where $n$ of these factors are associated with the default-free term structure. However, volatility sensitive instruments, such as interest rate derivatives and credit derivatives, will be affected by the $3 n$ factors associated with their volatilities. Thus of stochastic differential equations driven by correlated Wiener processes and express it as a system of stochastic differential equations driven by independent Wiener processes as presented in Assumption 2.1 The correlation between the short rate and the short-term credit spread shocks is denoted by $\rho_{i}^{f \lambda}$, the correlation between the short rate and volatility shocks is denoted by $\rho_{i}^{V f}$ and the correlation between the short-term credit spread and volatility shocks is denoted by $\rho_{i}^{V \lambda}$. 
the proposed model, subject to the specific correlation structure, allows for up to $n$ unspanned stochastic volatility factors that may affect only (interest rate and credit) derivative prices.

The absence of arbitrage implies that there exists an equivalent probability measure $\tilde{\mathbb{P}}$ where, for every maturity $T$, there is a $3 n$-dimensional process representing the market price of diffusion risk $\Phi(t)=\left\{\left(\phi_{1}(t), \phi_{2}(t), \ldots, \phi_{3 n}(t)\right), t \in[0, T]\right\}$, and a process $\psi(t)$ representing the market price of default risk such that $d \tilde{W}_{i}(t)=d W_{i}(t)-\phi_{i}(t) d t$, for $i=1,2, \ldots, 3 n$, is a $\tilde{\mathbb{P}}$-Wiener process and the default indicator process $N(t)$ has a $\tilde{\mathbb{P}}$-intensity $\tilde{h}(t)$, where $\tilde{h}(t)=\psi(t) h(t)$. Using Girsanov's theorem, and working along the lines of Heath et al. (1992) and Björk, Kabanov and Runggaldier (1997), the drift restriction for the HJM default-free forward rate and defaultable forward rate, respectively, are

$$
\begin{aligned}
& \alpha^{f}(t, T, \omega)=-\sum_{i=1}^{n} \sigma_{i}^{f}(t, T, \omega)\left(\phi_{i}(t)-\int_{t}^{T} \sigma_{i}^{f}(t, s, \omega) d s\right), \\
& \alpha^{d}(t, T, \omega)=-\sum_{i=1}^{2 n} \tilde{\sigma}_{i}^{d}(t, T, \omega)\left(\phi_{i}(t)-\int_{t}^{T} \tilde{\sigma}_{i}^{d}(t, s, \omega) d s\right)
\end{aligned}
$$

where $\alpha^{d}(t, T, \omega)=\alpha^{f}(t, T, \omega)+\alpha^{\lambda}(t, T, \omega)$ and $\tilde{\sigma}_{i}^{d}(t, T, \omega)=\tilde{\sigma}_{i}^{f}(t, T, \omega)+\tilde{\sigma}_{i}^{\lambda}(t, T, \omega)$. By combining (2.11) and (2.12), the credit spread drift is expressed in terms of the volatilities of the default-free forward rate and the credit spread as (recall that $\tilde{\sigma}_{i}^{f}(t, T, \omega)=$ $\sigma_{i}^{f}(t, T, \omega)$, for $i=1, \ldots, n$ and zero, otherwise)

$$
\begin{aligned}
\alpha^{\lambda}(t, T, \omega) & =-\sum_{i=1}^{2 n} \phi_{i}(t) \tilde{\sigma}_{i}^{\lambda}(t, T, \omega)+\sum_{i=1}^{2 n} \tilde{\sigma}_{i}^{\lambda}(t, T, \omega) \int_{t}^{T} \tilde{\sigma}_{i}^{\lambda}(t, s, \omega) d s \\
& +\sum_{i=1}^{n}\left(\tilde{\sigma}_{i}^{\lambda}(t, T, \omega) \int_{t}^{T} \sigma_{i}^{f}(t, s, \omega) d s+\sigma_{i}^{f}(t, T, \omega) \int_{t}^{T} \tilde{\sigma}_{i}^{\lambda}(t, s, \omega) d s\right) .
\end{aligned}
$$

By substituting the drift restrictions (2.11) and (2.13) into (2.4) and (2.5), respectively, we obtain the dynamics for the forward rate and forward credit spread processes under the risk-neutral measure as

$$
\begin{aligned}
& d f(t, T, \omega)=\sum_{i=1}^{n} \sigma_{i}^{f}(t, T, \omega) \int_{t}^{T} \sigma_{i}^{f}(t, s, \omega) d s d t+\sum_{i=1}^{n} \sigma_{i}^{f}(t, T, \omega) d \tilde{W}_{i}(t), \\
& d \lambda(t, T, \omega)=\sum_{i=1}^{2 n} \tilde{\sigma}_{i}^{\lambda}(t, T, \omega) \int_{t}^{T} \tilde{\sigma}_{i}^{\lambda}(t, s, \omega) d s d t+\sum_{i=1}^{n} \tilde{\sigma}_{i}^{\lambda}(t, T, \omega) \int_{t}^{T} \sigma_{i}^{f}(t, s, \omega) d s d t \\
& \quad+\sum_{i=1}^{n} \sigma_{i}^{f}(t, T, \omega) \int_{t}^{T} \tilde{\sigma}_{i}^{\lambda}(t, s, \omega) d s d t+\sum_{i=1}^{2 n} \tilde{\sigma}_{i}^{\lambda}(t, T, \omega) d \tilde{W}_{i}(t) .
\end{aligned}
$$


Further, by substituting the drift restriction (2.12) into (2.9), the risk-neutral dynamics of the defaultable forward rate are

$$
d f^{d}(t, T, \omega)=\sum_{i=1}^{2 n} \tilde{\sigma}_{i}^{d}(t, T, \omega)\left(\int_{t}^{T} \tilde{\sigma}_{i}^{d}(t, s, \omega) d s\right) d t+\sum_{i=1}^{2 n} \tilde{\sigma}_{i}^{d}(t, T, \omega) d \tilde{W}_{i}(t) .
$$

Accordingly the risk-neutral dynamics for the volatility processes $V_{i}(t), i=1, \ldots, n$ are

$$
\begin{aligned}
d V_{i}(t)= & {\left[\alpha_{i}^{V}\left(t, V_{i}\right)+\phi_{i}(t) z_{i}^{V_{1}} \sigma_{i 1}^{V}\left(t, V_{i}\right)+\phi_{n+i}(t) z_{i}^{V_{2}} \sigma_{i 2}^{V}\left(t, V_{i}\right)+\phi_{2 n+i}(t) z_{i}^{V_{3}} \sigma_{i 3}^{V}\left(t, V_{i}\right)\right] d t } \\
& +z_{i}^{V_{1}} \sigma_{i 1}^{V}\left(t, V_{i}\right) d \tilde{W}_{i}(t)+z_{i}^{V_{2}} \sigma_{i 2}^{V}\left(t, V_{i}\right) d \tilde{W}_{n+i}(t)+z_{i}^{V_{3}} \sigma_{i 3}^{V}\left(t, V_{i}\right) d \tilde{W}_{2 n+i}(t) .
\end{aligned}
$$

By integrating (2.14) and (2.15) and setting $T=t$, the risk-neutral dynamics of the short rate and short rate spread are 2

$$
\begin{aligned}
r(t, \omega) & =f(0, t)+\sum_{i=1}^{n} \int_{0}^{t} \sigma_{i}^{f}(u, t, \omega) \int_{u}^{t} \sigma_{i}^{f}(u, s, \omega) d s d u+\sum_{i=1}^{n} \int_{0}^{t} \sigma_{i}^{f}(u, t, \omega) d \tilde{W}_{i}(u), \\
c(t, \omega) & =\lambda(0, t)+\sum_{i=1}^{2 n} \int_{0}^{t} \tilde{\sigma}_{i}^{\lambda}(u, t, \omega) \int_{u}^{t} \tilde{\sigma}_{i}^{\lambda}(u, s, \omega) d s d u+\sum_{i=1}^{n} \int_{0}^{t} \tilde{\sigma}_{i}^{\lambda}(u, t) \int_{u}^{t} \sigma_{i}^{f}(u, s) d s d u \\
& +\sum_{i=1}^{n} \int_{0}^{t} \sigma_{i}^{f}(u, t, \omega) \int_{u}^{t} \tilde{\sigma}_{i}^{\lambda}(u, s, \omega) d s d u+\sum_{i=1}^{2 n} \int_{0}^{t} \tilde{\sigma}_{i}^{\lambda}(u, t, \omega) d \tilde{W}_{i}(u)
\end{aligned}
$$

Under general volatility functions, the default-free and the defaultable forward rate dynamics are typically Markovian in the entire yield curve, thereby leading to computational complexity when considering derivative pricing.

\section{A Markovian Defaultable Term Structure Model}

Motivated by Chiarella and Kwon (2001) and Björk et al. (2004), we propose next certain volatility structures that guarantee that the defaultable HJM term structure model admits finite dimensional realisations.

Assumption 3.1 The volatility functions are of the form

$$
\begin{array}{ll}
\sigma_{i}^{f}(t, T, \omega) & =\left[a_{0 i}+a_{1 i}(T-t)\right] \sqrt{r(t)} \sqrt{V_{i}(t)} e^{-\kappa_{i}^{f}(T-t)}, \quad 1 \leq i \leq n \\
\sigma_{i}^{\lambda}(t, T, \omega) & =\left[b_{0 i}+b_{1 i}(T-t)\right] \sqrt{c(t)} \sqrt{V_{i}(t)} e^{-\kappa_{i}^{\lambda}(T-t)}, \quad 1 \leq i \leq 2 n
\end{array}
$$

where $\kappa_{i}^{f}, \kappa_{i}^{\lambda}, a_{0 i}, a_{1 i}, b_{0 i}$ and $b_{1 i}$ are constants.

This class of volatility functions gives rise to a high degree of flexibility in modelling the wide range of shapes of the yield curve by virtue of the polynomial in the deterministic

\footnotetext{
${ }^{2}$ For notational convenience we set $f(0, t):=f(0, t, \omega)$ and $\lambda(0, t):=\lambda(0, t, \omega)$.
} 
part. These volatility specifications are level dependent, allow for hump-shaped shocks and entail unspanned stochastic volatility. We remind the reader that the dependence on the default-free short rate, the short-term credit spread and volatility is represented by $\omega \in \Omega$.

Proposition 3.1 Under the volatility structure of Assumption 3.1, the default-free forward rate and the forward credit spread are respectively expressed a. 3

$$
\begin{aligned}
& f(t, T, \omega)=f(0, T)+\sum_{i=1}^{n} B_{x_{1 i}}(T-t) x_{1 i}(t)+\sum_{i=1}^{n} \sum_{j=1}^{6} B_{\Phi_{j i}}(T-t) \Phi_{j i}(t), \\
& \lambda(t, T, \omega)=\lambda(0, T)+\sum_{i=1}^{n} \sum_{j=2}^{3} B_{x_{j i}}(T-t) x_{j i}(t)+\sum_{i=1}^{n} \sum_{j=7}^{20} B_{\Phi_{j i}}(T-t) \Phi_{j i}(t) .
\end{aligned}
$$

Thus, the defaultable forward rate $f^{d}(t, T, \omega)$ is expressed as

$$
f^{d}(t, T, \omega)=f^{d}(0, T)+\sum_{i=1}^{n} \sum_{j=1}^{3} B_{x_{j i}}(T-t) x_{j i}(t)+\sum_{i=1}^{n} \sum_{j=1}^{20} B_{\Phi_{j i}}(T-t) \Phi_{j i}(t),
$$

where

$$
\left\{\begin{aligned}
B_{x_{1 i}}(T-t)= & {\left[a_{0 i}+a_{1 i}(T-t)\right] e^{-\kappa_{i}^{f}(T-t)}, } \\
B_{x_{2 i}}(T-t)= & \rho_{i}^{f \lambda}\left[b_{0 i}+b_{1 i}(T-t)\right] e^{-\kappa_{i}^{\lambda}(T-t)}, \\
B_{x_{3 i}}(T-t)= & \sqrt{1-\left(\rho_{i-n}^{\lambda f}\right)^{2}}\left[b_{0 i}+b_{1 i}(T-t)\right] e^{-\kappa_{i}^{\lambda}(T-t)}, \\
B_{\Phi_{1 i}}(T-t)= & a_{1 i} e^{-\kappa_{i}^{f}(T-t)}, \\
B_{\Phi_{2 i}}(T-t)= & \frac{a_{1 i}}{\kappa_{i}^{f}}\left(\frac{1}{\kappa_{i}^{f}}+\frac{a_{0 i}}{a_{1 i}}\right)\left[a_{0 i}+a_{1 i}(T-t)\right] e^{-\kappa_{i}^{f}(T-t),} \\
B_{\Phi_{3 i}}(T-t)= & -\left[\frac{a_{1 i} a_{1 i}}{\kappa_{i}^{f}}\left(\frac{1}{\kappa_{i}^{f}}+\frac{a_{0 i}}{a_{1 i}}\right)\right. \\
& \left.+\frac{a_{1 i}}{\kappa_{i}^{f}}\left(\frac{a_{1 i}}{\kappa_{i}^{f}}+2 a_{0 i}\right)(T-t)+\frac{\left(a_{1 i}\right)^{2}}{\kappa_{i}^{f}}(T-t)^{2}\right] e^{-2 \kappa_{i}^{f}(T-t)}, \\
B_{\Phi_{4 i}}(T-t)= & \frac{\left(a_{1 i}\right)^{2}}{\kappa_{i}^{f}}\left(\frac{1}{\kappa_{i}^{f}}+\frac{a_{0 i}}{a_{1 i}}\right) e^{-\kappa_{i}^{f}(T-t)}, \\
B_{\Phi_{5 i}}(T-t)= & -\frac{a_{1 i}}{\kappa_{i}^{f}}\left[\frac{a_{1 i}}{\kappa_{i}^{f}}+2 a_{0 i}+2 a_{1 i}(T-t)\right] e^{-2 \kappa_{i}^{f}(T-t),} \\
B_{\Phi_{6 i}}(T-t)= & -\frac{\left(a_{1 i}\right)^{2}}{\kappa_{i}^{f}} e^{-2 \kappa_{i}^{f}(T-t)},
\end{aligned}\right.
$$

\footnotetext{
${ }^{3}$ For notational convenience for all $t \in[0, T]$, we set $f(0, t):=f(0, t, \omega)$ and $\lambda(0, t):=\lambda(0, t, \omega)$.
} 


$$
\left\{\begin{aligned}
B_{\Phi_{7 i}}(T-t)= & \rho_{i}^{f \lambda}\left[\frac{a_{0 i} b_{0 i}}{\kappa_{i}^{f}}+\frac{a_{1 i} b_{0 i}}{\left(\kappa_{i}^{f}\right)^{2}}+\left(\frac{a_{0 i} b_{1 i}}{\kappa_{i}^{f}}+\frac{a_{1 i} b_{1 i}}{\left(\kappa_{i}^{f}\right)^{2}}\right)(T-t)\right] e^{-\kappa_{i}^{\lambda}(T-t)}, \\
B_{\Phi_{8 i}}(T-t)=- & \rho_{i}^{f \lambda}\left[\frac{a_{0 i} b_{0 i}}{\kappa_{i}^{f}}+\frac{a_{1 i} b_{0 i}}{\left(\kappa_{i}^{f}\right)^{2}}+\left(\frac{a_{1 i} b_{0 i}}{\kappa_{i}^{f}}+\frac{a_{0 i} b_{1 i}}{\kappa_{i}^{f}}+\frac{a_{1 i} b_{1 i}}{\left(\kappa_{i}^{f}\right)^{2}}\right)(T-t)\right. \\
& \left.\quad+\frac{a_{1 i} b_{1 i}}{\kappa_{i}^{f}}(T-t)^{2}\right] e^{-\left(\kappa_{i}^{f}+\kappa_{i}^{\lambda}\right)(T-t)}, \\
B_{\Phi_{9 i}}(T-t)= & \rho_{i}^{f \lambda}\left(\frac{a_{0 i} b_{0 i}}{\kappa_{i}^{f}}+\frac{a_{1 i} b_{1 i}}{\left(\kappa_{i}^{f}\right)^{2}}\right) e^{-\kappa_{i}^{\lambda}(T-t)}, \\
B_{\Phi_{10 i}}(T-t)= & -\rho_{i}^{f \lambda}\left[\frac{a_{1 i} b_{0 i}}{\kappa_{i}^{f}}+\frac{a_{0 i} b_{1 i}}{\kappa_{i}^{f}}+\frac{a_{1 i} b_{1 i}}{\left(\kappa_{i}^{f}\right)^{2}}-2 \frac{a_{1 i} b_{1 i}}{\kappa_{i}^{f}}(T-t)\right] e^{-\left(\kappa_{i}^{f}+\kappa_{i}^{\lambda}\right)(T-t)} \\
B_{\Phi_{11 i}}(T-t)= & -\rho_{i}^{f \lambda} \frac{a_{11} b_{1 i}}{\kappa_{i}^{f}} e^{-\left(\kappa_{i}^{f}+\kappa_{i}^{\lambda}\right)(T-t)}, \\
B_{\Phi_{12 i}}(T-t)= & \rho_{i}^{f \lambda}\left[\frac{a_{0 i} b_{0 i}}{\kappa_{i}^{\lambda}}+\frac{a_{0 i} b_{1 i}}{\left(\kappa_{i}^{\lambda}\right)^{2}}+\left(\frac{a_{1 i} b_{0 i}}{\kappa_{i}^{\lambda}}+\frac{a_{1 i} b_{1 i}}{\left(\kappa_{i}^{\lambda}\right)^{2}}\right)(T-t)\right] e^{-\kappa_{i}^{f}(T-t)} \\
B_{\Phi_{13 i}}(T-t)= & \rho_{i}^{f \lambda}\left(\frac{a_{0 i} b_{0 i}}{\kappa_{i}^{\lambda}}+\frac{a_{1 i} b_{1 i}}{\left(\kappa_{i}^{\lambda}\right)^{2}}\right) e^{-\kappa_{i}^{f}(T-t)},
\end{aligned}\right.
$$

$$
\left\{\begin{array}{l}
B_{\Phi_{14 i}}(T-t)=\frac{b_{1 i}}{\kappa_{i}^{\lambda}}\left(\frac{1}{\kappa_{i}^{\lambda}}+\frac{b_{0 i}}{b_{1 i}}\right)\left[b_{0 i}+b_{1 i}(T-t)\right] e^{-\kappa_{i}^{\lambda}(T-t)}, \\
B_{\Phi_{15 i}}(T-t)=\left[\frac{b_{1 i} b_{1 i}}{\kappa_{i}^{\lambda}}\left(\frac{1}{\kappa_{i}^{\lambda}}+\frac{b_{0 i}}{b_{1 i}}\right)+\frac{b_{1 i}}{\kappa_{i}^{\lambda}}\left(\frac{b_{1 i}}{\kappa_{i}^{\lambda}}+2 b_{0 i}\right)(T-t)+\frac{\left(b_{1 i}\right)^{2}}{\kappa_{i}^{\lambda}}(T-t)^{2}\right] e^{-2 \kappa_{i}^{\lambda}(T-t)}, \\
B_{\Phi_{16 i}}(T-t)=\frac{\left(b_{1 i}\right)^{2}}{\kappa_{i}^{\lambda}}\left(\frac{1}{\kappa_{i}^{\lambda}}+\frac{b_{0 i}}{b_{1 i}}\right) e^{-\kappa_{i}^{\lambda}(T-t)}, \\
B_{\Phi_{17 i}}(T-t)=-\frac{b_{1 i}}{\kappa_{i}^{\lambda}}\left[\frac{b_{1 i}}{\kappa_{i}^{\lambda}}+2 b_{0 i}+2 b_{1 i}(T-t)\right] e^{-2 \kappa_{i}^{\lambda}(T-t)}, \\
B_{\Phi_{18 i}}(T-t)=-\frac{\left(b_{1 i}\right)^{2}}{\kappa_{i}^{\lambda}} e^{-2 \kappa_{i}^{\lambda}(T-t)}, \\
B_{\Phi_{19 i}}(T-t)=\rho_{i}^{f \lambda} b_{1 i} e^{-\kappa_{i}^{\lambda}(T-t)}, \\
B_{\Phi_{20 i}}(T-t)=\sqrt{1-\left(\rho_{i-n}^{\lambda f}\right)^{2}} b_{1 i} e^{-\kappa_{i}^{\lambda}(T-t)},
\end{array}\right.
$$

and the state variables $x_{j i}(t)$ and $\Phi_{j i}(t)$ satisfy the stochastic and ordinary differential equations given in Corollary 3.2.

Proof: See Appendix A for technical details.

Corollary 3.2 The state variables $x_{j i}(t)$ and $\Phi_{j i}(t)$ satisfy the stochastic differential 
equations

$$
\left\{\begin{array}{l}
d x_{1 i}(t)=-\kappa_{i}^{f} x_{1 i}(t) d t+\sqrt{r(t) V_{i}(t)} d \tilde{W}_{i}(t), \\
d x_{2 i}(t)=-\kappa_{i}^{\lambda} x_{2 i}(t) d t+\sqrt{c(t) V_{i}(t)} d \tilde{W}_{i}(t), \\
d x_{3 i}(t)=-\kappa_{i}^{\lambda} x_{3 i}(t) d t+\sqrt{c(t) V_{i}(t)} d \tilde{W}_{n+i}(t), \\
d \Phi_{1 i}(t)=\left[x_{1 i}(t)-\kappa_{i}^{f} \Phi_{1 i}(t)\right] d t, \quad d \Phi_{2 i}(t)=\left[r(t) V_{i}(t)-\kappa_{i}^{f} \Phi_{2 i}(t)\right] d t, \\
d \Phi_{3 i}(t)=\left[r(t) V_{i}(t)-2 \kappa_{i}^{f} \Phi_{3 i}(t)\right] d t, \quad d \Phi_{4 i}(t)=\left[\Phi_{2 i}(t)-\kappa_{i}^{f} \Phi_{4 i}(t)\right] d t, \\
d \Phi_{5 i}(t)=\left[\Phi_{3 i}(t)-2 \kappa_{i}^{f} \Phi_{5 i}(t)\right] d t, \quad d \Phi_{6 i}(t)=\left[2 \Phi_{5 i}(t)-2 \kappa_{i}^{f} \Phi_{6 i}(t)\right] d t, \\
d \Phi_{7 i}(t)=\left[V_{i}(t) \sqrt{r(t) c(t)}-\kappa_{i}^{\lambda} \Phi_{7 i}(t)\right] d t, \quad d \Phi_{8 i}(t)=\left[V_{i}(t) \sqrt{r(t) c(t)}-\left(\kappa_{i}^{f}+\kappa_{i}^{\lambda}\right) \Phi_{8 i}(t)\right] d t, \\
d \Phi_{9 i}(t)=\left[\Phi_{9 i}(t)-\kappa_{i}^{\lambda} \Phi_{9 i}(t)\right] d t, \quad d \Phi_{10 i}(t)=\left[\Phi_{10 i}(t)-\left(\kappa_{i}^{f}+\kappa_{i}^{\lambda}\right) \Phi_{10 i}(t)\right] d t, \\
d \Phi_{11 i}(t)=\left[2 \Phi_{12 i}(t)-\left(\kappa_{i}^{f}+\kappa_{i}^{\lambda}\right) \Phi_{11 i}(t)\right] d t, \quad d \Phi_{12 i}(t)=\left[V_{i}(t) \sqrt{r(t) c(t)}-\kappa_{i}^{f} \Phi_{12 i}(t)\right] d t, \\
d \Phi_{13 i}(t)=\left[\Phi_{14 i}(t)-\kappa_{i}^{f} \Phi_{13 i}(t)\right] d t, \quad d \Phi_{14 i}(t)=\left[c(t) V_{i}(t)-\kappa_{i}^{\lambda} \Phi_{14 i}(t)\right] d t, \\
d \Phi_{15 i}(t)=\left[c(t) V_{i}(t)-2 \kappa_{i}^{\lambda} \Phi_{15 i}(t)\right] d t, \quad d \Phi_{16 i}(t)=\left[\Phi_{16 i}(t)-\kappa_{i}^{\lambda} \Phi_{16 i}(t)\right] d t, \\
d \Phi_{17 i}(t)=\left[\Phi_{15 i}(t)-2 \kappa_{i}^{\lambda} \Phi_{17 i}(t)\right] d t, \quad d \Phi_{18 i}(t)=\left[2 \Phi_{17 i}(t)-2 \kappa_{i}^{\lambda} \Phi_{18 i}(t)\right] d t, \\
d \Phi_{19 i}(t)=\left[x_{2 i}(t)-\kappa_{i}^{\lambda} \Phi_{19 i}(t)\right] d t, \quad d \Phi_{20 i}(t)=\left[x_{3 i}(t)-\kappa_{i}^{\lambda} \Phi_{20 i}(t)\right] d t,
\end{array}\right.
$$

subject to the initial conditions $x_{j i}(0)=\Phi_{j i}(0)=0$ for $i=1, \ldots, n$ and $j=1, \ldots, 20$.

Proof: Take the stochastic differential of (A.4), (A.10) and (A.12) in Appendix A to obtain the stochastic differential equations.

Note that the system in Corollary 3.2 needs to be augmented by the stochastic differential equations for the volatility functions $V_{i}(t)$ that satisfy the SDEs (2.17) as well as the Markovian representation for the short rate and short term credit spread given in the following colollary.

Corollary 3.3 The short rate and the short term credit spread can be expressed as

$$
\begin{aligned}
& r(t, \omega)=f(0, t)+\sum_{i=1}^{n} \alpha_{1 i} x_{1 i}(t)+\sum_{i=1}^{n} \sum_{j=1}^{6} \beta_{j i} \Phi_{j i}(t), \\
& c(t, \omega)=\lambda(0, t)+\sum_{i=1}^{n} \sum_{j=2}^{3} \alpha_{j i} x_{j i}(t)+\sum_{i=1}^{n} \sum_{j=7}^{20} \beta_{j i} \Phi_{j i}(t),
\end{aligned}
$$

where $\alpha_{j i}=B_{x_{j i}}(0)$ and $\beta_{j i}=B_{\Phi_{j i}}(0)$.

Proof: Setting $T=t$ in (3.3) and (3.4) derives the above representations. 
Proposition 3.4 The default-free bond price $P(t, T, \omega)$ and the defaultable bond price $P^{d}(t, T)$ are both exponential affine with

$$
\begin{aligned}
P(t, T, \omega) & =\frac{P(0, T)}{P(0, t)} \exp \left(-\sum_{i=1}^{n} D_{x_{1 i}}(T-t) x_{1 i}(t)-\sum_{i=1}^{n} \sum_{j=1}^{6} D_{\Phi_{j i}}(T-t) \Phi_{j i}(t)\right), \\
P^{d}(t, T, \omega) & =\frac{P^{d}(0, T)}{P^{d}(0, t)} \exp \left(-\sum_{i=1}^{n} \sum_{j=1}^{3} D_{x_{j i}}(T-t) x_{j i}(t)-\sum_{i=1}^{n} \sum_{j=1}^{20} D_{\Phi_{j i}}(T-t) \Phi_{j i}(t)\right),
\end{aligned}
$$

respectively, where $x_{j i}(t)$ and $\Phi_{j i}(t)$ are specified in Corollary 3.2. The deterministic functions $D_{x_{j i}}(T-t)$ and $D_{\Phi_{j i}}(T-t)$ are given by

$$
\left\{\begin{array}{l}
D_{x_{1 i}}(T-t)=\frac{1}{\left(\kappa_{i}^{f}\right)^{2}}\left[a_{0 i} \kappa_{i}^{f}+a_{1 i}-e^{-\kappa_{i}^{f}(T-t)}\left(a_{0 i} \kappa_{i}^{f}+a_{1 i}+a_{1 i} \kappa_{i}^{f}(T-t)\right)\right], \\
D_{x_{2 i}}(T-t)=\frac{\rho_{i}^{f \lambda}}{\left(\kappa_{i}^{\lambda}\right)^{2}}\left[a_{0 i} \kappa_{i}^{\lambda}+a_{1 i}-e^{-\kappa_{i}^{\lambda}(T-t)}\left(a_{0 i} \kappa_{i}^{\lambda}+a_{1 i}+a_{1 i} \kappa_{i}^{\lambda}(T-t)\right)\right], \\
D_{x_{3 i}}(T-t)=\frac{\sqrt{1-\left(\rho_{i-n}^{\lambda f}\right)^{2}}}{\left(\kappa_{i}^{\lambda}\right)^{2}}\left[a_{0 i} \kappa_{i}^{\lambda}+a_{1 i}-e^{-\kappa_{i}^{\lambda}(T-t)}\left(a_{0 i} \kappa_{i}^{\lambda}+a_{1 i}+a_{1 i} \kappa_{i}^{\lambda}(T-t)\right)\right],
\end{array}\right.
$$

$$
\left\{\begin{aligned}
D_{\Phi_{1 i}}(T-t) & =\frac{a_{1 i}}{\kappa_{i}^{f}}\left(1-e^{-\kappa_{i}^{f}(T-t)}\right), \\
D_{\Phi_{2 i}}(T-t) & =\left(\frac{a_{1 i}}{\kappa_{i}^{f}}\right)^{2}\left(\frac{1}{\kappa_{i}^{f}}+\frac{a_{0 i}}{a_{1 i}}\right)\left[\left(\frac{1}{\kappa_{i}^{f}}+\frac{a_{0 i}}{a_{1 i}}\right)\left(e^{-\kappa_{i}^{f}(T-t)}-1\right)+(T-t) e^{-\kappa_{i}^{f}(T-t)}\right], \\
D_{\Phi_{3 i}}(T-t) & =-\left(\frac{a_{1 i}}{\left(\kappa_{i}^{f}\right)^{2}}\right)\left[\left(\frac{a_{1 i}}{2\left(\kappa_{i}^{f}\right)^{2}}+\frac{a_{0 i}}{\kappa_{i}^{f}}+\frac{\left(a_{0 i}\right)^{2}}{2 a_{1 i}}\right)\left(e^{-2 \kappa_{i}^{f}(T-t)}-1\right)\right. \\
& +\left(\frac{a_{1 i}}{\kappa_{i}^{f}}+a_{0 i}\right)(T-t) e^{-2 \kappa_{i}^{f}(T-t)}+\frac{a_{1 i}}{2}(T-t)^{2} e^{\left.-2 \kappa_{i}^{f}(T-t)\right]} \\
D_{\Phi_{4 i}}(T-t) & =\left(\frac{a_{1 i}}{\kappa_{i}^{f}}\right)^{2}\left(\frac{1}{\kappa_{i}^{f}}+\frac{a_{0 i}}{a_{1 i}}\right)\left(e^{-\kappa_{i}^{f}(T-t)}-1\right), \\
D_{\Phi_{5 i}}(T-t) & =-\left(\frac{a_{1 i}}{\left(\kappa_{i}^{f}\right)^{2}}\right)\left[\left(\frac{a_{1 i}}{\kappa_{i}^{f}}+a_{0 i}\right)\left(e^{-2 \kappa_{i}^{f}(T-t)}-1\right)+a_{1 i}(T-t) e^{\left.-2 \kappa_{i}^{f}(T-t)\right]}\right. \\
D_{\Phi_{6 i}}(T-t) & =-\frac{1}{2}\left(\frac{a_{1 i}}{\kappa_{i}^{f}}\right)^{2}\left(e^{-2 \kappa_{i}^{f}(T-t)}-1\right),
\end{aligned}\right.
$$

\footnotetext{
${ }^{4}$ For notational convenience we set for all $t \in[0, T], P(0, t):=P(0, t, \omega)$ and $P^{d}(0, t):=\bar{P}^{d}(0, t, \omega)$.
} 


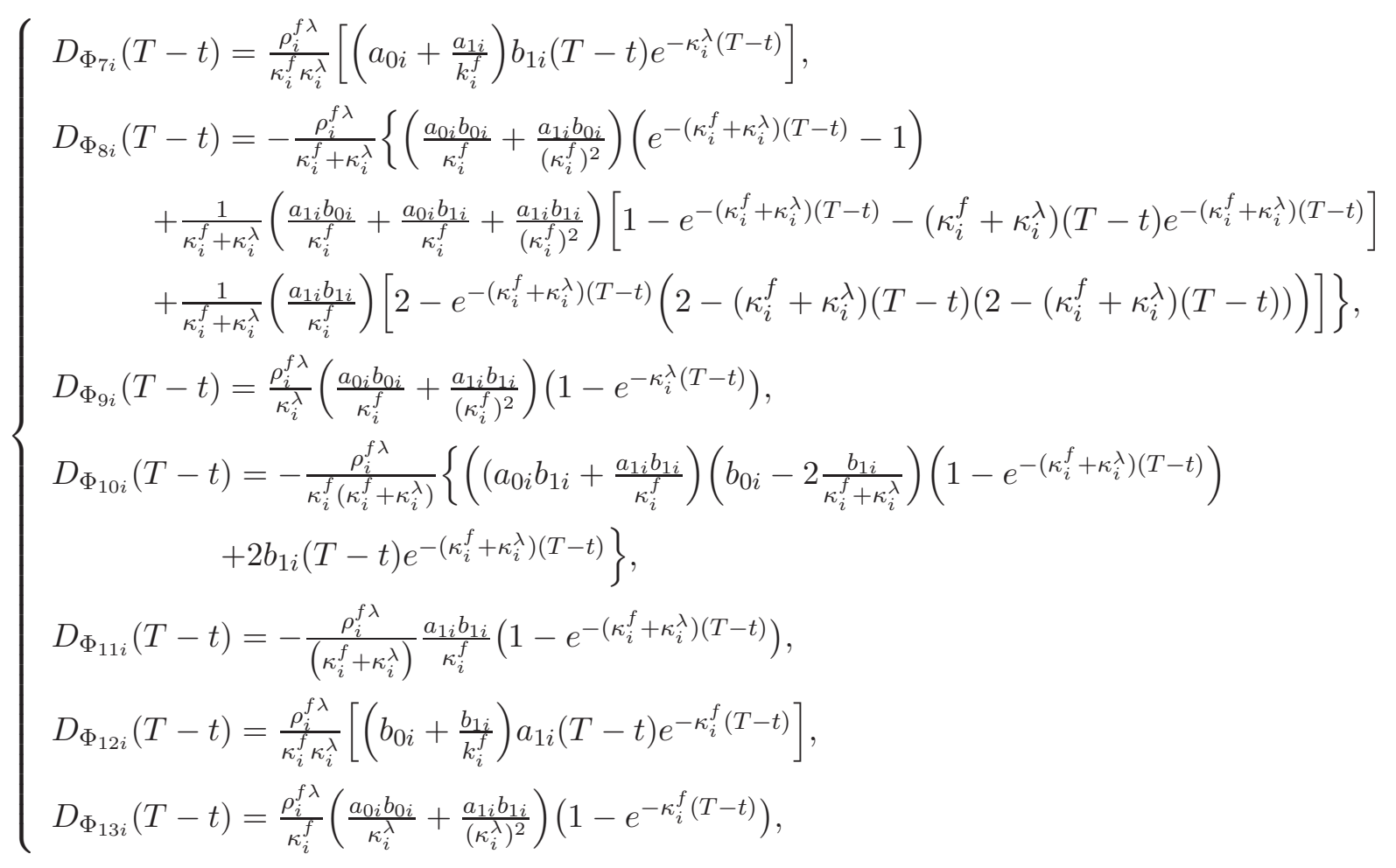

and finally we have

$$
\left\{\begin{aligned}
D_{\Phi_{14 i}}(T-t) & =\left(\frac{b_{1 i}}{\kappa_{i}^{\lambda}}\right)^{2}\left(\frac{1}{\kappa_{i}^{\lambda}}+\frac{b_{0 i}}{b_{1 i}}\right)\left[\left(\frac{1}{\kappa_{i}^{\lambda}}+\frac{b_{0 i}}{b_{1 i}}\right)\left(e^{-\kappa_{i}^{\lambda}(T-t)}-1\right)+(T-t) e^{\left.-\kappa_{i}^{\lambda}(T-t)\right]}\right. \\
D_{\Phi_{15 i}}(T-t) & =-\left(\frac{b_{1 i}}{\left(\kappa_{i}^{\lambda}\right)^{2}}\right)\left[\left(\frac{b_{1 i}}{2\left(\kappa_{i}^{\lambda}\right)^{2}}+\frac{b_{0 i}}{\kappa_{i}^{\lambda}}+\frac{\left(b_{0 i}\right)^{2}}{2 b_{1 i}}\right)\left(e^{-2 \kappa_{i}^{\lambda}(T-t)}-1\right)\right. \\
+ & \left(\frac{b_{1 i}}{\kappa_{i}^{\lambda}}+b_{0 i}\right)(T-t) e^{-2 \kappa_{i}^{\lambda}(T-t)}+\frac{b_{1 i}}{2}(T-t)^{2} e^{\left.-2 \kappa_{i}^{\lambda}(T-t)\right]}, \\
D_{\Phi_{16 i}}(T-t) & =\left(\frac{b_{1 i}}{\kappa_{i}^{\lambda}}\right)^{2}\left(\frac{1}{\kappa_{i}^{\lambda}}+\frac{b_{0 i}}{b_{1 i}}\right)\left(e^{-\kappa_{i}^{\lambda}(T-t)}-1\right), \\
D_{\Phi_{17 i}}(T-t) & =-\left(\frac{b_{1 i}}{\left(\kappa_{i}^{\lambda}\right)^{2}}\right)\left[\left(\frac{b_{1 i}}{\kappa_{i}^{\lambda}}+b_{0 i}\right)\left(e^{-2 \kappa_{i}^{\lambda}(T-t)}-1\right)+b_{1 i}(T-t) e^{\left.-2 \kappa_{i}^{\lambda}(T-t)\right]},\right. \\
D_{\Phi_{18 i}}(T-t) & =-\frac{1}{2}\left(\frac{b_{1 i}}{\kappa_{i}^{\lambda}}\right)^{2}\left(e^{-2 \kappa_{i}^{\lambda}(T-t)}-1\right), \quad D_{\Phi_{19 i}}(T-t)=\frac{\rho_{i}^{f \lambda} b_{1 i}}{\kappa_{i}^{\lambda}}\left(1-e^{-\kappa_{i}^{\lambda}(T-t)}\right), \\
D_{\Phi_{20 i}}(T-t) & =\sqrt{1-\left(\rho_{i-n}^{\lambda f}\right)^{2}} \frac{b_{1 i}}{\kappa_{i}^{\lambda}}\left(1-e^{-\kappa_{i}^{\lambda}(T-t)}\right) .
\end{aligned}\right.
$$

Proof: See Appendix B for the technical details.

Proposition 3.4 is the main theoretical contribution of the paper. The default-free bond price can be expressed in terms of $8 n(=7 n+n)$ state variables while the defaultable bond price is expressed in terms of $24 n(=23 n+n)$ state variables, where the $n$ state variables are associated with $n$ stochastic volatility variables. Both default-free and defaultable bond prices are exponential affine functions of these state variables and the $7 n$ state variables driving the default-free bond prices constitute a common set for both 
default-free and defaultable bond prices. Even though the dimension of the state space is relatively large, the driving sources of uncertainty of the entire state space is only $3 n$.

The proposed model can be easily adjusted to accommodate exponentially decaying volatility functional forms for the forward rates and credit spreads resulting in a Markovian system with a lower dimension state space as has been shown in Chiarella, Maina and Nikitopoulos-Sklibosios (2010). Finite dimensional realisations can also be obtained by expressing the state variables as a linear combination of fixed tenor forward rates, along the lines of Chiarella and Kwon (2003) and Chiarella et al. (2007).

\section{Pricing of Credit Default Swaps and Swaptions}

By using the proposed defaultable term structure model, we derive pricing formulas for single-name credit default swaps (CDS hereafter) and credit default swaptions in the absence of counterparty risk 5

\subsection{Credit Default Swaps}

A CDS contract with maturity $T$ allows the insured party to receive protection, up to time $T$, from the insurer against default of the reference obligor. When the default time $\tau$ is $t<\tau \leq T$, then the insurer makes the protection payment $(1-\mathcal{R})$ at $\tau$, where $\mathcal{R}$ is the recovery rate. Then at $t<\tau$, the value of the protection leg is

$$
W_{p r t}(t)=e^{-\int_{t}^{\tau} r(s) d s}(1-\mathcal{R}) \mathbb{1}_{\{t<\tau \leq T\}}
$$

The insured party pays the premium $\pi$ at times $t_{i}, i=1,2, \ldots, N$ until either the contract maturity $t_{N}=T$, if no default occurs, or until default, if $\tau \leq T$. By denoting as $\delta_{i}=t_{i-1}-t_{i}$, then the value at time $t<t_{1}$ of the premium leg, including the accrual payment for the fraction of time in which default occurs, is given by

$$
W_{\text {prm }}(t)=\pi \sum_{i=1}^{N} \delta_{i} e^{-\int_{t}^{t_{i}} r(s) d s} \mathbb{1}_{\left\{\tau>t_{i}\right\}}+\pi\left(\tau-t_{\tau-1}\right) e^{-\int_{t}^{\tau} r(s) d s} \mathbb{1}_{\{t<\tau<T\}},
$$

where $t_{1}$ is the first premium payment date and $t_{\tau-1}$ is the last premium payment date $t_{i}$ before the default time $\tau$. Then, the value of the CDS can be expressed under the risk-neutral probability measure as

$$
C D S_{\pi}(t)=\tilde{\mathbb{E}}\left[W_{p r t}(t)-W_{p r m}(t) \mid \mathcal{F}_{t}\right]
$$

\footnotetext{
${ }^{5}$ The possible extension to incorporate counterparty risk is discussed in Chiarella et al. (2011).
} 
The fair premium rate $\tilde{\pi}(t)$, the so called CDS spread, is the rate that will make the value of the CDS equal to zero. Working along the lines of Brigo and Morini (2005)6 for a contract that settles immediately on default of the reference obligor, the CDS spread is given by

$$
\tilde{\pi}(t)=\frac{\mathbb{1}_{\{\tau>t\}}(1-\mathcal{R}) \int_{t}^{T} \tilde{\mathbb{E}}\left[\tilde{h}(u) e^{-\int_{t}^{u}(r(s)+\tilde{h}(s)) d s} \mid \mathcal{F}_{t}^{W}\right] d u}{\mathbb{1}_{\{\tau>t\}} \sum_{i=1}^{N} \delta_{i} \bar{P}^{d}\left(t, t_{i}\right)+\tilde{\mathbb{E}}\left[\left(\tau-t_{\tau-1}\right) e^{-\int_{t}^{\tau} r(s) d s} \mathbb{1}_{\{t<\tau<T\}} \mid \mathcal{F}_{t}\right]} .
$$

Instead of the protection payment to be made at default time $\tau$, the protection payment could be deferred to the first premium payment date $t_{i}$ following default time $\left(t_{i}>\tau\right)$. This gives rise to the postponed running CDS whose main advantage is that the absence of accrued-interest term in $\left(\tau-t_{\tau-1}\right)$ ensures that all payments occur at the canonical grid of the $t_{i}$ 's. We then have the following result.

Corollary 4.1 When the protection payment is postponed to the first premium payment date $t_{i}$ following the default time, the CDS spread can be approximated by

$$
\tilde{\pi}(t) \approx \frac{(1-\mathcal{R}) \sum_{i=1}^{N}\left[\bar{P}^{d}\left(t, t_{i-1}\right)-\bar{P}^{d}\left(t, t_{i}\right)\right]}{\sum_{i=1}^{N}\left(t_{i}-t_{i-1}\right) \bar{P}^{d}\left(t, t_{i}\right)} .
$$

Proof: Work along the lines of Brigo and Morini (2005).

\subsection{Credit Default Swaptions}

Credit default swaptions (hereafter CDS options) are options written on CDS contracts. More precisely, a plain-vanilla CDS option with maturity $T_{m}$ is a European option on a forward credit default swap (hereafter forward CDS). The underlying forward CDS is a CDS contract issued at time $s$ with a start date $T_{m}$ and maturity $T$, with $0 \leq s \leq T_{m}<T 7$ This contract gives default protection over the future interval $\left[T_{m}, T\right]$ but if the reference obligor defaults before the start date, this is $\tau<T_{m}$, the contract is terminated and no payments are made. By an appropriate choice of numeraire that depends on the value of the premium leg and the survival process $\mathbb{Q}\left(t<\tau \mid \mathcal{F}_{t}^{N}\right)$, Rutkowski and Armstrong (2009) define an equivalent probability measure $\overline{\mathcal{Q}}$, and show that the price of the CDS option can be expressed as

$$
\mathcal{C}_{\text {swpt }}(t)=\mathbb{1}_{\{t<\tau\}} \tilde{A}(t) \overline{\mathbb{E}}\left[\left(\tilde{\pi}_{f}\left(t, T_{m}\right)-K\right)^{+} \mid \mathcal{F}_{t}^{W}\right]
$$

\footnotetext{
${ }^{6} \mathrm{~A}$ detailed proof of the result is presented in the working paper version of the current paper, see Chiarella et al. (2011).

${ }^{7}$ See Bielecki, Jeanblanc and Rutkowski (2007) for a formal definition.
} 
where $\tilde{\pi}_{f}\left(t, T_{m}\right)$ is the forward CDS spread, $\overline{\mathbb{E}}$ is the expectation under the $\overline{\mathcal{Q}}$ measure and

$$
\tilde{A}(t)=\mathbb{Q}\left(t<\tau \mid \mathcal{F}_{t}^{W}\right)^{-1} \tilde{\mathbb{E}}\left[\bar{W}_{p r m}(t) \mid \mathcal{F}_{t}^{W}\right] .
$$

The forward CDS spread is an $\left(\mathcal{F}^{W}, \overline{\mathcal{Q}}\right)$-martingale and its dynamics follow the driftless stochastic differential equation

$$
d \tilde{\pi}_{f}\left(t, T_{m}\right)=\bar{\sigma}(t) \tilde{\pi}_{f}\left(t, T_{m}\right) d \bar{W}(t)
$$

where $\bar{W}$ is a $\overline{\mathcal{Q}}$-Brownian motion. 8

Proposition 4.2 The value of a credit default swaption with strike $K$ and maturity $T_{m}$ can be calculated by the Black's formula

$$
\mathcal{C}_{\text {swpt }}(t)=\mathbb{1}_{\{t<\tau\}} \tilde{A}(t)\left[\tilde{\pi}_{f}\left(t, T_{m}\right) N\left(d_{1}\right)-K N\left(d_{2}\right)\right]
$$

where

$$
d_{1}=\frac{\ln \left(\frac{\tilde{\pi}_{f}\left(t, T_{m}\right)}{K}\right)+\frac{\bar{\sigma}^{2}}{2}\left(T_{m}-t\right)}{\bar{\sigma} \sqrt{T_{m}-t}} \quad \text { and } \quad d_{2}=d_{1}-\bar{\sigma} \sqrt{T_{m}-t}
$$

and $\bar{\sigma}$ is constant for different tenor dates.

Proof: Work along the lines of Rutkowski and Armstrong (2009) 9

Note that $\bar{\sigma}$ is the only parameter to be inferred from market data. Although the model is not easily calibrated to quoted data if the market is illiquid, it provides a platform where prices of different options can be translated into implied volatilities thereby giving more information on the market.

\section{$5 \quad$ Numerical Study}

In this section, we conduct a sensitivity analysis to investigate the impact of the model parameters including correlations and stochastic volatility, on bond prices, credit default swap rates and the value of credit default swaptions.

\footnotetext{
${ }^{8}$ We assume that $\tilde{\pi}_{f}(t)$ follows lognormal dynamics. It was remarked in Brigo and Morini (2005) that this distributional assumption is inspired by standard models used to model equity and interest rate markets. Jabbour, El-Masri and Young (2008) however rejected this hypothesis by showing that the log forward CDS spreads exhibit large positive skewness and excess kurtosis.

${ }^{9}$ For completeness, we prove the result in the working paper version of the current paper, see Chiarella et al. (2011).
} 
We assume that the initial term structures of forward rate and forward credit spread are given by

$$
f(0, T)=0.04-0.04 \sqrt{V(0)} e^{-0.18 T} \text { and } \lambda(0, T)=0.04-0.01 \sqrt{V(0)} e^{-0.1 T}
$$

respectively, with the initial volatility chosen to be $V(0)=1.00$. We consider the case of $n=1$ and we specify for $i=1,2,3$ the market price of risk $\phi_{i}(t)=\bar{\phi}_{i} \sqrt{V_{1}(t)}$ with the scaling factor $\bar{\phi}_{i}=1$. In (2.17), we further assume that $\alpha_{1}^{V}\left(t, V_{1}\right)=\kappa_{V}\left(\bar{V}-V_{1}(t)\right)$ and $\sigma_{1}^{V}\left(t, V_{1}\right)=\bar{\sigma}_{1}^{V} \sqrt{V_{1}(t)}$ such that

$$
d V_{1}(t)=\left[\kappa^{V} \bar{V}-\left(\kappa^{V}-\sum_{j=1}^{3} z_{1}^{V_{j}} \bar{\sigma}_{1}^{V}\right) V_{1}(t)\right] d t+\bar{\sigma}_{1}^{V} \sum_{j=1}^{3} z_{1}^{V_{j}} \sqrt{V_{1}(t)} d \tilde{W}_{j}(t),
$$

where $z_{1}^{V_{j}}$ is given in (2.8). Table 1 specifies the parameters values used in the analysis.

\begin{tabular}{cccccccccccc}
$a_{01}$ & $a_{11}$ & $b_{01}$ & $b_{11}$ & $\bar{V}$ & $\bar{\sigma}_{1}^{V}$ & $\kappa_{V}$ & $\kappa_{f}$ & $\kappa_{\lambda}$ & $\rho^{V \lambda}$ & $\rho^{f V}$ & $\rho^{f \lambda}$ \\
\hline 0.135 & 0.035 & 0.145 & 0.0045 & 0.7542 & 1.5 & 2.1 & 0.30 & 0.40 & 0.25 & 0.44 & -0.40
\end{tabular}

Table 1: Parameter values. These are the parameter values used in the numerical analysis undertaken in this section, unless stated otherwise.

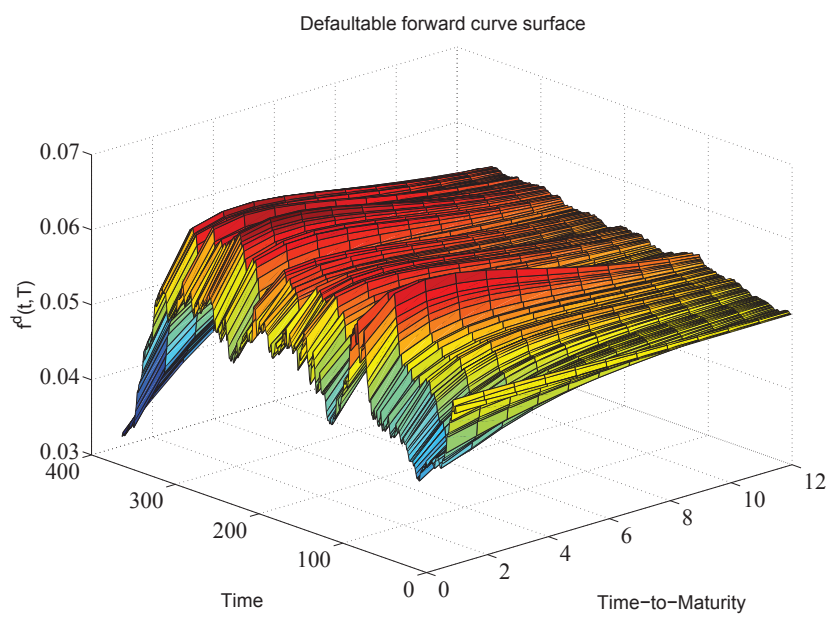

Figure 1: Defaultable Forward Rate Curve. A possible evolution of the defaultable forward rate curve surface produced by simulating (3.5), for $0 \leq t \leq 400$. The time to maturity is expressed in years. Clearly the proposed model is capable of generating a variety of shapes for the defaultable forward rate curve. 
Figure1 1llustrates a possible evolution of the defaultable forward rate curve surface (3.5) for $0 \leq t \leq 400$. As it is clear in Figure 1 the proposed hump-shaped level dependent stochastic volatility model can generate a variety of shapes for the defaultable forward rate curve.

\subsection{Bond Price Distributions}

The defaultable bond price is exponentially affine as presented in (3.12). Figure 2 illustrates the impact of the various correlations, namely $\rho^{V \lambda}, \rho^{f V}, \rho^{f \lambda}$, as well as the volatility parameter $\bar{\sigma}_{1}^{V}$ on the simulated distribution of the pseudo bond price with maturity $T=1$.

When the correlation between the short-term credit spread and volatility, $\rho^{V \lambda}$, is increased (holding the other correlations constant) the distribution of the bond price skews to the right, see Figure 2(a). This increment is more pronounced when the correlation between the short rate and volatility, $\rho^{f V}$, is increased, see Figure 2(b), since the short rate process, in our case, has higher average volatility than the short term credit spread. Increasing the correlation between the short rate and short term credit spread, $\rho^{f \lambda}$, decreases the kurtosis of the bond price distribution, as Figure 2(c) depicts. In Figure $2(\mathrm{~d})$ we observe that increasing $\bar{\sigma}_{1}^{V}$, which affects the volatility of the volatility, skews the distribution to the right, a feature that is consistent with the empirical evidence by D'Souza et al. (2004).

\subsection{Sensitivity Analysis - Credit Default Swaps}

The shape of the credit curves is influenced by the demand and supply for credit protection in the CDS market and reflects the credit quality of the reference entities. By using the parameter values of Table 1, unless otherwise stated, we perform a numerical study to gauge the effect of the correlations and the stochastic volatility to the CDS spreads computed by (4.5).

Table 2 depicts the effect of increasing maturity on the CDS spread (4.5), given a recovery rate of $40 \%, 10$ for varying values of correlations $\rho^{f \lambda}$ and $\rho^{f V}$. Although Krekel and Wenzel (2006) and Berndt et al. (2010) argued that the effect of the correlation $\rho^{f \lambda}$ is not very significant and need not be taken into consideration when calculating CDS spreads, our results suggest that it has an impact on the CDS premium. We observe that varying the correlation $\rho^{f \lambda}$ from negative to positive leads to an increase

\footnotetext{
${ }^{10}$ We adopt recovery assumptions as proposed in Pan and Singleton (2008).
} 


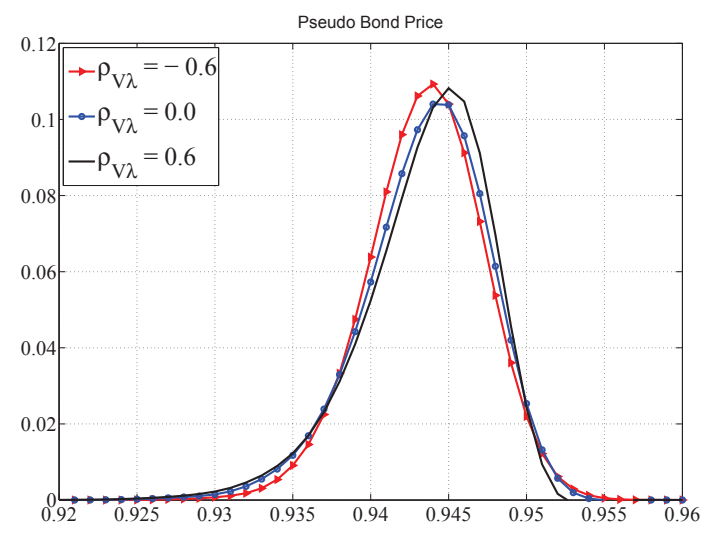

(a)

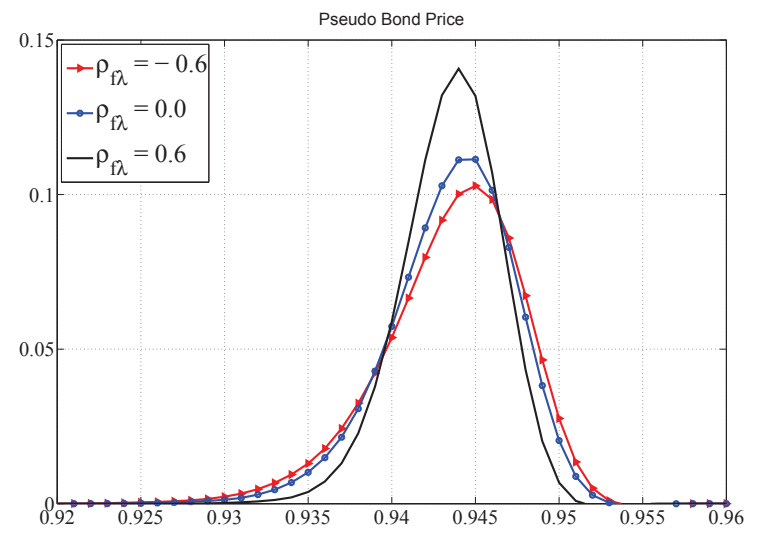

(c)

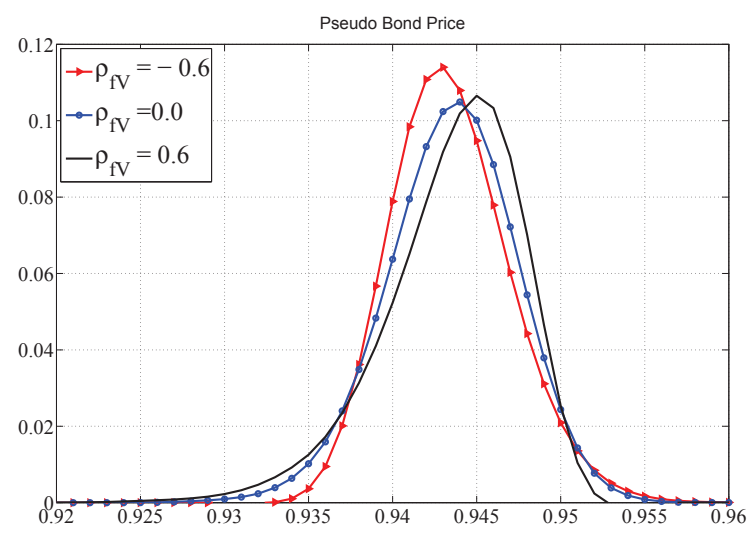

(b)

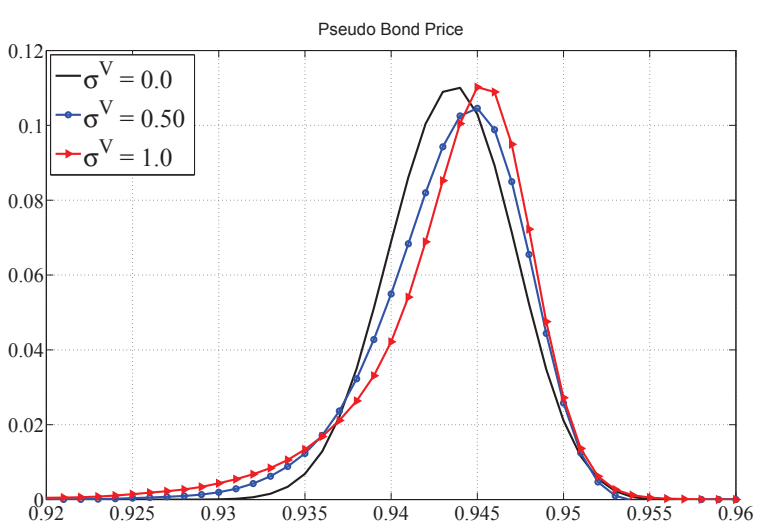

(d)

Figure 2: Distribution of the Defaultable Bond Prices (3.12). Panel (a) depicts the distribution of the defaultable bond prices, when the correlation $\rho^{V \lambda}$ between the short-term credit spread and volatility takes the values -0.6,0,0.6. Panel (b) depicts the distribution of the defaultable bond prices, when the correlation $\rho^{f V}$ between the short default-free rate and volatility takes the values -0.6,0,0.6. Panel (c) depicts the distribution of the defaultable bond prices, when the correlation $\rho^{f \lambda}$ between the short default-free rate and short-term credit spread takes the values -0.6,0,0.6. Panel (d) depicts the distribution of the defaultable bond prices, when the scaling factor of the volatility of volatility $\sigma^{V} \equiv \bar{\sigma}_{1}^{V}$ takes the values $0,0.50,1.0$. Increasing values of $\rho^{V \lambda}, \rho^{f V}$ and $\bar{\sigma}_{1}^{V}$ skew the distribution of the bond price to the right, while increasing values of $\rho^{f \lambda}$ decrease the kurtosis of the bond price distribution. The maturity of the defaultable bond is one year. "Pseudo" bond refers to a defaultable bond that has not defaulted up to current time. 
in the CDS spread, while varying the correlation $\rho^{f V}$ from negative to positive leads to a decrease in the CDS spread. The CDS spread curve exhibits a hump-shaped structure for varying maturities. During nonvolatile market conditions, the cost of protection over the longer term is usually higher as it is difficult to predict cash flows and future events that effect the profitability of a firm over a longer period thereby yielding an increasing CDS curve. Additionally a decrease in $\bar{\sigma}_{1}^{V}$ (that affects the volatility of volatility) leads to an increase in the CDS spread, see Table 2 .

\subsection{Sensitivity Analysis - Credit Default Swaptions}

To illustrate the model's properties we compute the swaption prices with varying parameters. At time $t=0$, we compute the price of the swaption according to (4.9) with maturity $T_{m}=0.5$ issued on a credit default swap that has a defaultable bond (based on the framework developed in Section 3) with a maturity of $T=2$ years as its underlying and the default protection is required for the period $[0.5,2.5]$. In addition, we use the following parameters for the simulation: recovery rate $\mathcal{R}=40 \%$, volatility of the forward CDS rate $\bar{\sigma}=0.411$ with $N=400$.

Figure 3 gives the credit default swaption price under varying strike rates and correlations $\rho^{f \lambda}$ and $\rho^{f V}$. We observe that increasing $K$ leads to a decrease in the credit default swaption rate which is in line with the market behavior that deep in-the-money options trade higher than at-the-money and out-of-the-money options. Negative correlation $\rho^{f \lambda}$ produces lower swaption prices, while negative correlation $\rho^{f V}$ produces higher swaption prices.

From Figure 4, we observe that an increase in $\bar{\sigma}_{1}^{V}$ leads to a decrease in the swaption prices, with the effect being more pronounced for deep in the money options. This is consistent with the results in Table 2 as an increasing $\bar{\sigma}_{1}^{V}$ leads to a reduction in the CDS rates. Furthermore, as the time to maturity increases, the credit default swaption becomes more sensitive to changes in the volatility specifications.

\footnotetext{
${ }^{11}$ It was noted in Schönbucher (2004) that the value of the volatility $\tilde{\pi}_{f}=0.4$ is of an acceptable level for simulation purposes.
} 

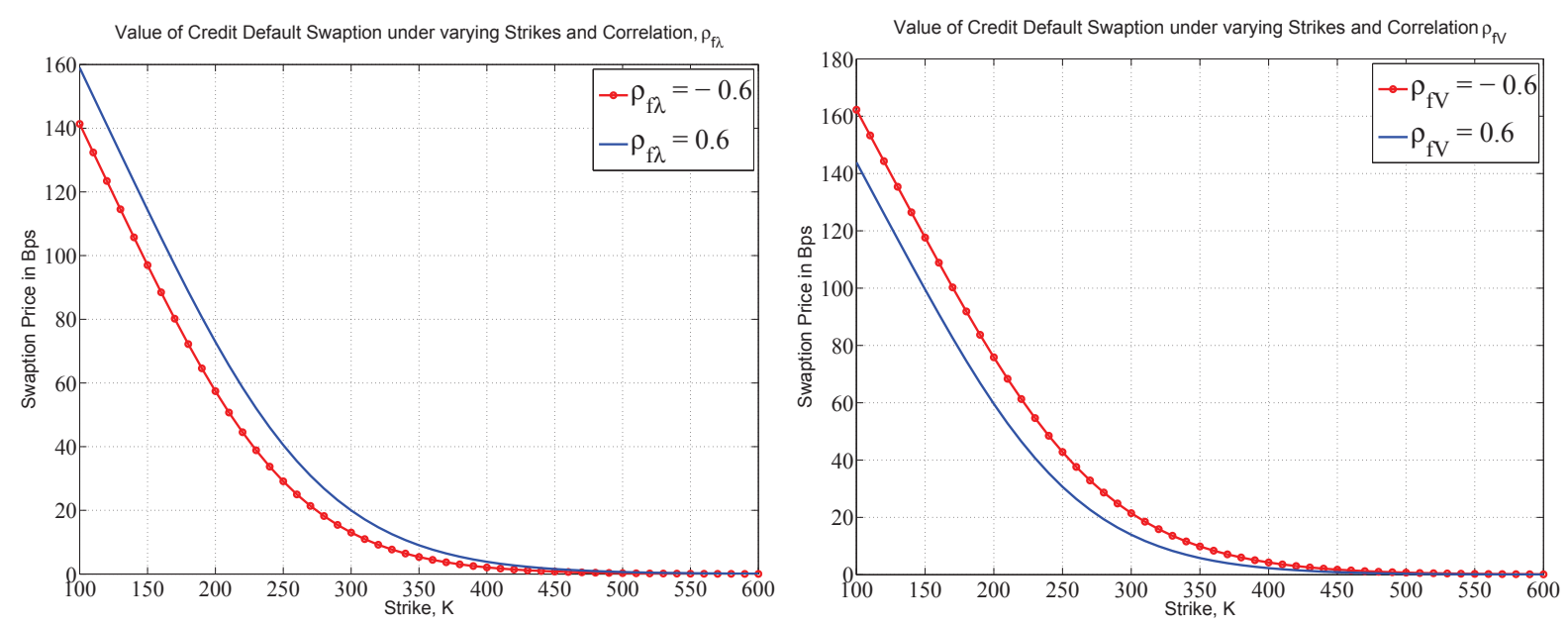

Figure 3: Simulated Credit Default Swaption Prices. The graph displays simulated credit default swaption prices (in bps) computed by (4.9) for varying strikes and correlations $\rho^{f \lambda}$ and $\rho^{f V}$. The credit default swaption matures in $T_{m}=0.5$ years and it is issued on a credit default swap with maturity $T=2$ years. Increasing strikes decrease the credit default swaption prices, while, increasing values of $\rho^{f \lambda}$ and $\rho^{f V}$ lead to an increase in the swaption prices and a decrease in the swaption prices, respectively.
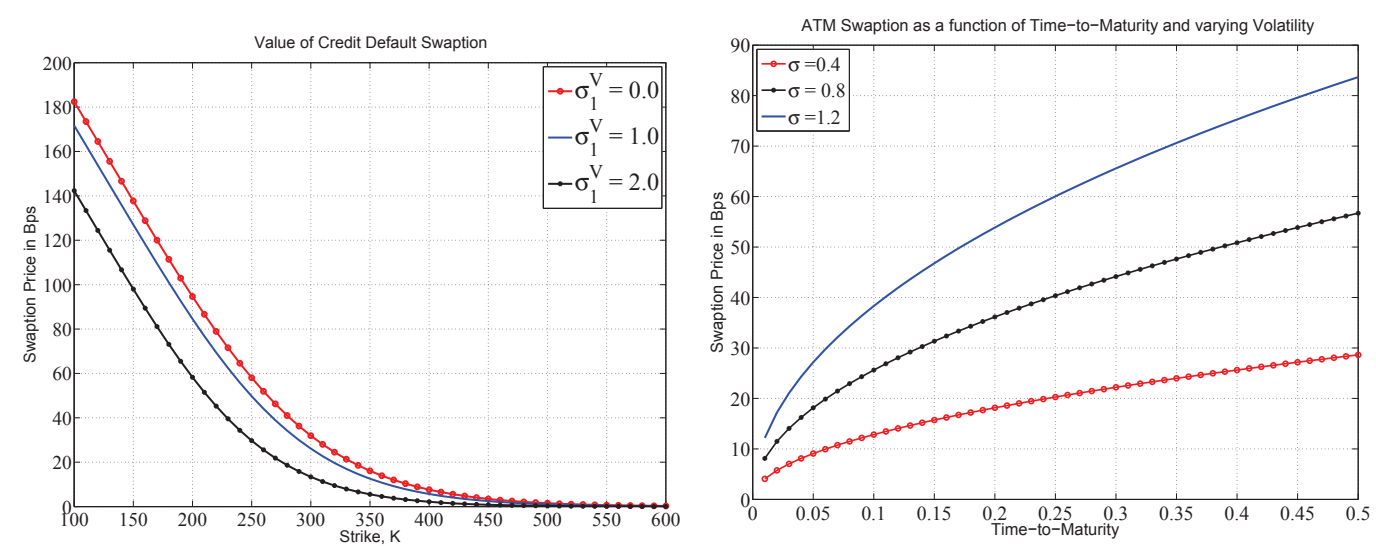

Figure 4: Credit Default Swaption Prices and Volatility. The graph displays simulated credit default swaption prices (in bps) computed by (4.9) for varying strikes, parameter values of $\bar{\sigma}_{1}^{V}$ (that affects the volatility of volatility) and time to maturity. The credit default swaption matures in $T_{m}=0.5$ years and it is issued on a credit default swap with maturity $T=2$ years. Increasing strikes decrease the credit default swaption prices, while, an increase in $\bar{\sigma}_{1}^{V}$ leads to a decrease in the swaption prices. Furthermore, as the time to maturity increases, ATM credit default swaption becomes more sensitive to changes in the volatility specifications. 


\begin{tabular}{|c|c|c|c|c|c|c|c|c|c|c|c|c|}
\hline $\mathrm{T}$ & & $\rho^{f \lambda}$ & & & & $\rho^{f V}$ & & & & $\bar{\sigma}_{1}^{V}$ & & \\
\hline rears) & -0.6 & -0.3 & 0.3 & 0.6 & -0.6 & -0.3 & 0.3 & 0.6 & 0.0 & 0.5 & 1.0 & 1.5 \\
\hline 1 & 243.627 & 245.129 & 247.128 & 248.123 & 247.113 & 246.113 & 244.622 & 244.627 & 248.615 & 248.123 & 247.123 & 244.627 \\
\hline 2 & 261.390 & 268.311 & 278.414 & 281.303 & 285.0323 & 281.834 & 269.153 & 264.291 & 287.651 & 286.500 & 281.871 & 265.717 \\
\hline 3 & 256.657 & 266.245 & 284.564 & 290.608 & 305.702 & 300.015 & 270.737 & 257.990 & 307.872 & 305.983 & 295.740 & 262.497 \\
\hline 4 & 246.106 & 260.409 & 287.853 & 296.186 & 321.064 & 314.099 & 267.892 & 247.343 & 323.527 & 320.828 & 306.055 & 255.134 \\
\hline 5 & 232.332 & 249.513 & 285.050 & 297.202 & 331.499 & 323.352 & 261.221 & 232.332 & 334.467 & 331.280 & 312.130 & 243.162 \\
\hline
\end{tabular}

Table 2: Simulated CDS Spreads. The table reports simulated CDS spreads (in bps) computed by (4.5) for varying maturities, correlations $\rho^{f \lambda}$ and $\rho^{f V}$, as well as, parameter values of $\bar{\sigma}_{1}^{V}$ (that affects the volatility of volatility). Increasing values of $\rho^{f \lambda}$ and $\rho^{f V}$ lead to an increase in the CDS spread and a decrease in the CDS spread, respectively. Increasing values of $\bar{\sigma}_{1}^{V}$ lead to a decrease in the CDS spread. The CDS spread curve exhibits a hump-shaped structure for increasing maturities. 


\section{Acknowledgements}

We thank seminar participants at the 2009 Quantitative Methods in Finance and the UTS Finance Discipline Group internal research seminars for fruitful discussions and helpful suggestions. The authors also gratefully acknowledge useful comments from anonymous referees. The usual caveat applies.

\section{Appendix}

\section{A Proof of Proposition 3.1}

We recall from (2.14) that under the risk-neutral measure, the dynamics of the default-free forward rate can be written as

$f(t, T)=f(0, T)+\sum_{i=1}^{n} \int_{0}^{t} \sigma_{i 1}^{f}\left(u, T, V_{i}\right) \int_{u}^{T} \sigma_{i 1}^{f}\left(u, s, V_{i}\right) d s d u+\sum_{i=1}^{n} \int_{0}^{t} \sigma_{i 1}^{f}\left(u, T, V_{i}\right) d \tilde{W}_{i}(u)$.

Using the volatility specifications (3.1), the stochastic integral equation (A.1) becomes

$$
\begin{aligned}
f(t, T)=f & (0, T)+\sum_{i=1}^{n} \int_{0}^{t} r(u) V_{i}(u)\left[a_{0 i}+a_{1 i}(T-u)\right] e^{-\kappa_{i}^{f}(T-u)} \int_{u}^{T}\left[a_{0 i}+a_{1 i}(s-u)\right] e^{-\kappa_{i}^{f}(s-u)} d s d u \\
& +\sum_{i=1}^{n} \int_{0}^{t} \sqrt{r(u) V_{i}(u)}\left[a_{0 i}+a_{1 i}(T-u)\right] e^{-\kappa_{i}^{f}(T-u)} d \tilde{W}_{i}(u) .
\end{aligned}
$$

By using the property $T-u=(T-t)+(t-u)$, and performing standard algebraic manipulations, equation (A.2) becomes

$$
\begin{aligned}
f(t, T) & =f(0, T)+\sum_{i=1}^{n} \frac{a_{1 i}}{\kappa_{i}^{f}}\left(\frac{1}{\kappa_{i}^{f}}+\frac{a_{0 i}}{a_{1 i}}\right)\left[a_{0 i}+a_{1 i}(T-t)\right] e^{-\kappa_{i}^{f}(T-t)} \Phi_{2 i}(t) \\
& +\sum_{i=1}^{n}\left[\frac{a_{1 i} a_{1 i}}{\kappa_{i}^{f}}\left(\frac{1}{\kappa_{i}^{f}}+\frac{a_{0 i}}{a_{1 i}}\right)+\frac{a_{1 i}}{\kappa_{i}^{f}}\left(\frac{a_{1 i}}{\kappa_{i}^{f}}+2 a_{0 i}\right)(T-t)+\frac{a_{1 i}}{\kappa_{i}^{f}}(T-t)^{2}\right] e^{-\kappa_{i}^{f}(T-t)} \Phi_{3 i}(t) \\
& +\sum_{i=1}^{n} \frac{\left(a_{1 i}\right)^{2}}{\kappa_{i}^{f}}\left(\frac{1}{\kappa_{i}^{f}}+\frac{a_{0 i}}{a_{1 i}}\right) e^{-\kappa_{i}^{f}(T-t)} \Phi_{4 i}(t) \\
& -\sum_{i=1}^{n} \frac{a_{1 i}}{\kappa_{i}^{f}}\left[\frac{a_{1 i}}{\kappa_{i}^{f}}+2 a_{0 i}+2 a_{1 i}(T-t)\right] e^{-2 \kappa_{i}^{f}(T-t)} \Phi_{5 i}(t)-\sum_{i=1}^{n} \frac{\left(a_{1 i}\right)^{2}}{\kappa_{i}^{f}} e^{-2 \kappa_{i}^{f}(T-t)} \Phi_{6 i}(t) \\
& +\sum_{i=1}^{n}\left[a_{0 i}+a_{1 i}(T-t)\right] e^{-\kappa_{i}^{f}(T-t)} x_{1 i}(t)+\sum_{i=1}^{n} a_{1 i} e^{-\kappa_{i}^{f}(T-t)} \Phi_{1 i}(t),
\end{aligned}
$$


where

$$
\left\{\begin{array}{l}
x_{1 i}(t)=\int_{0}^{t} \sqrt{r(u) V_{i}(u)} e^{-\kappa_{i}^{f}(t-u)} d \tilde{W}_{i}(u), \\
\Phi_{1 i}(t)=\int_{0}^{t} \sqrt{r(u) V_{i}(u)}(t-u) e^{-\kappa_{i}^{f}(t-u)} d \tilde{W}_{i}(u), \\
\Phi_{2 i}(t)=\int_{0}^{t} r(u) V_{i}(u) e^{-\kappa_{i}^{f}(t-u)} d u \\
\Phi_{3 i}(t)=\int_{0}^{t} r(u) V_{i}(u) e^{-2 \kappa_{i}^{f}(t-u)} d u \\
\Phi_{4 i}(t)=\int_{0}^{t} r(u) V_{i}(u)(t-u) e^{-\kappa_{i}^{f}(t-u)} d u, \\
\Phi_{5 i}(t)=\int_{0}^{t} r(u) V_{i}(u)(t-u) e^{-2 \kappa_{i}^{f}(t-u)} d u, \\
\Phi_{6 i}(t)=\int_{0}^{t} r(u) V_{i}(u)(t-u)^{2} e^{-2 \kappa_{i}^{f}(t-u)} d u,
\end{array}\right.
$$

Thus, the forward rate process is Markovian and more specifically is affine in the state space variables since

$$
f(t, T)=f(0, T)+\sum_{i=1}^{n} B_{x_{1 i}}(T-t) x_{1 i}(t)+\sum_{i=1}^{n} \sum_{j=1}^{6} B_{\Phi_{j i}}(T-t) \Phi_{j i}(t),
$$

where $B_{x_{1 i}}(T-t)$ and $B_{\Phi_{j i}}(T-t)$ are given in (3.6).

Similarly, we recall from (2.15) that the forward credit spread dynamics under the risk-neutral measure can be written as

$$
\begin{aligned}
\lambda(t, T) & =\sum_{i=1}^{2 n} \int_{0}^{t} \tilde{\sigma}_{i}^{\lambda}(u, T) \int_{u}^{T} \tilde{\sigma}_{i}^{\lambda}(u, s) d s d u+\sum_{i=1}^{n} \int_{0}^{t}\left(\tilde{\sigma}_{i}^{\lambda}(u, T) \int_{u}^{T} \sigma_{i}^{f}(u, s) d s+\sigma_{i}^{f}(u, T) \int_{u}^{T} \tilde{\sigma}_{i}^{\lambda}(u, s) d s\right) d u \\
& +\sum_{i=1}^{2 n} \int_{0}^{t} \tilde{\sigma}_{i}^{\lambda}(u, T) d \tilde{W}_{i}(u) .
\end{aligned}
$$

Then by using the volatility specifications of Assumption 3.1, the drift components in the forward credit spread representation (A.6) are given by

$$
\begin{aligned}
& \sum_{i=1}^{n} \int_{0}^{t} \tilde{\sigma}_{i}^{\lambda}(u, T) \int_{u}^{T} \sigma_{i}^{f}(u, s) d s d u \\
& \quad=\sum_{i=1}^{n} \rho_{i}^{f \lambda}\left\{\left[\frac{a_{0 i} b_{0 i}}{\kappa_{i}^{f}}+\frac{a_{1 i} b_{0 i}}{\left(\kappa_{i}^{f}\right)^{2}}+\left(\frac{a_{0 i} b_{1 i}}{\kappa_{i}^{f}}+\frac{a_{1 i} b_{1 i}}{\left(\kappa_{i}^{f}\right)^{2}}\right)(T-t)\right] e^{-\kappa_{i}^{\lambda}(T-t)} \Phi_{7 i}(t)\right. \\
& \quad-\left[\frac{a_{0 i} b_{0 i}}{\kappa_{i}^{f}}+\frac{a_{1 i} b_{0 i}}{\left(\kappa_{i}^{f}\right)^{2}}+\left(\frac{a_{1 i} b_{0 i}}{\kappa_{i}^{f}}+\frac{a_{0 i} b_{1 i}}{\kappa_{i}^{f}}+\frac{a_{1 i} b_{1 i}}{\left(\kappa_{i}^{f}\right)^{2}}\right)(T-t)+\frac{a_{1 i} b_{1 i}}{\kappa_{i}^{f}}(T-t)^{2}\right] e^{-\left(\kappa_{i}^{f}+\kappa_{i}^{\lambda}\right)(T-t)} \Phi_{8 i}(t) \\
& \quad+\left(\frac{a_{0 i} b_{0 i}}{\kappa_{i}^{f}}+\frac{a_{1 i} b_{1 i}}{\left(\kappa_{i}^{f}\right)^{2}}\right) e^{-\kappa_{i}^{\lambda}(T-t)} \Phi_{9 i}(t) \\
& \quad-\left[\frac{a_{1 i} b_{0 i}}{\kappa_{i}^{f}}+\frac{a_{0 i} b_{1 i}}{\kappa_{i}^{f}}+\frac{a_{1 i} b_{1 i}}{\left(\kappa_{i}^{f}\right)^{2}}-2 \frac{a_{1 i} b_{1 i}}{\kappa_{i}^{f}}(T-t)\right] e^{-\left(\kappa_{i}^{f}+\kappa_{i}^{\lambda}\right)(T-t)} \Phi_{10 i}(t) \\
& \left.\left.\quad-\frac{a_{1 i} b_{1 i}}{\kappa_{i}^{f}} e^{-\left(\kappa_{i}^{f}+\kappa_{i}^{\lambda}\right)(T-t)} \Phi_{11 i}(t)\right)\right\},
\end{aligned}
$$


and

$$
\begin{aligned}
& \sum_{i=1}^{n} \int_{0}^{t} \sigma_{i}^{f}(u, T) \int_{u}^{T} \tilde{\sigma}_{i}^{\lambda}(u, s) d s d u=\sum_{i=1}^{n} \rho_{i}^{f \lambda}\{ \\
& \quad-\left[\frac{a_{0 i} b_{0 i}}{\kappa_{i}^{\lambda}}+\frac{a_{0 i} b_{1 i}}{\left(\kappa_{i}^{\lambda}\right)^{2}}+\left(\frac{a_{0 i} b_{1 i}}{\kappa_{i}^{\lambda}}+\frac{a_{1 i} b_{0 i}}{\kappa_{i}^{\lambda}}+\frac{a_{1 i} b_{1 i}}{\left(\kappa_{i}^{\lambda}\right)^{2}}\right)(T-t)+\frac{a_{1 i} b_{1 i}}{\kappa_{i}^{\lambda}}(T-t)^{2}\right] e^{-\left(\kappa_{i}^{f}+\kappa_{i}^{\lambda}\right)(T-t)} \Phi_{8 i}(t) \\
& \quad-\left[\frac{b_{1 i} a_{0 i}}{\kappa_{i}^{\lambda}}+\frac{b_{0 i} a_{1 i}}{\kappa_{i}^{\lambda}}+\frac{a_{1 i} b_{1 i}}{\left(\kappa_{i}^{\lambda}\right)^{2}}-2 \frac{a_{1 i} b_{1 i}}{\kappa_{i}^{\lambda}}(T-t)\right] e^{-\left(\kappa_{i}^{f}+\kappa_{i}^{\lambda}\right)(T-t)} \Phi_{10 i}(t) \\
& \quad-\frac{a_{1 i} b_{1 i}}{\kappa_{i}^{\lambda}} e^{-\left(\kappa_{i}^{f}+\kappa_{i}^{\lambda}\right)(T-t)} \Phi_{11 i}(t)+\left[\frac{a_{0 i} b_{0 i}}{\kappa_{i}^{\lambda}}+\frac{a_{0 i} b_{1 i}}{\left(\kappa_{i}^{\lambda}\right)^{2}}+\left(\frac{a_{1 i} b_{0 i}}{\kappa_{i}^{\lambda}}+\frac{a_{1 i} b_{1 i}}{\left(\kappa_{i}^{\lambda}\right)^{2}}\right)(T-t)\right] e^{-\kappa_{i}^{f}(T-t)} \Phi_{12 i}(t) \\
& +
\end{aligned}
$$

and finally

$$
\begin{aligned}
& \sum_{i=1}^{2 n} \int_{0}^{t} \tilde{\sigma}_{i}^{\lambda}(u, T) \int_{u}^{T} \tilde{\sigma}_{i}^{\lambda}(u, s) d s d u=\sum_{i=1}^{n}\left\{\frac{b_{1 i}}{\kappa_{i}^{\lambda}}\left(\frac{1}{\kappa_{i}^{\lambda}}+\frac{b_{0 i}}{b_{1 i}}\right)\left[b_{0 i}+b_{1 i}(T-t)\right] e^{-\kappa_{i}^{\lambda}(T-t)} \Phi_{14 i}(t)\right. \\
& \quad+\left[\frac{b_{1 i} b_{1 i}}{\kappa_{i}^{\lambda}}\left(\frac{1}{\kappa_{i}^{\lambda}}+\frac{b_{0 i}}{b_{1 i}}\right)+\frac{b_{1 i}}{\kappa_{i}^{\lambda}}\left(\frac{b_{1 i}}{\kappa_{i}^{\lambda}}+2 b_{0 i}\right)(T-t)+\frac{b_{1 i}}{\kappa_{i}^{\lambda}}(T-t)^{2}\right] e^{-\kappa_{i}^{\lambda}(T-t)} \Phi_{15 i}(t) \\
& \quad+\frac{\left(b_{1 i}\right)^{2}}{\kappa_{i}^{\lambda}}\left(\frac{1}{\kappa_{i}^{\lambda}}+\frac{b_{0 i}}{b_{1 i}}\right) e^{-\kappa_{i}^{\lambda}(T-t)} \Phi_{16 i}(t) \\
& \left.\quad-\frac{b_{1 i}}{\kappa_{i}^{\lambda}}\left[\frac{b_{1 i}}{\kappa_{i}^{\lambda}}+2 b_{0 i}+2 b_{1 i}(T-t)\right] e^{-2 \kappa_{i}^{\lambda}(T-t)} \Phi_{17 i}(t)-\frac{\left(b_{1 i}\right)^{2}}{\kappa_{i}^{\lambda}} e^{-2 \kappa_{i}^{\lambda}(T-t)} \Phi_{18 i}(t)\right\},
\end{aligned}
$$

where we introduce the additional state variables

$$
\left\{\begin{array}{l}
\Phi_{7 i}(t)=\int_{0}^{t} \eta_{i}(u) e^{-\kappa_{i}^{\lambda}(t-u)} d u, \quad \Phi_{8 i}(t)=\int_{0}^{t} \eta_{i}(u) e^{-\left(\kappa_{i}^{f}+\kappa_{i}^{\lambda}\right)(t-u)} d u, \\
\Phi_{9 i}(t)=\int_{0}^{t} \eta_{i}(u)(t-u) e^{-\kappa_{i}^{\lambda}(t-u)} d u, \quad \Phi_{10 i}(t)=\int_{0}^{t} \eta_{i}(u)(t-u) e^{-\left(\kappa_{i}^{f}+\kappa_{i}^{\lambda}\right)(t-u)} d u, \\
\Phi_{11 i}(t)=\int_{0}^{t} \eta_{i}(u)(t-u)^{2} e^{-\left(\kappa_{i}^{f}+\kappa_{i}^{\lambda}\right)(t-u)} d u, \quad \Phi_{12 i}(t)=\int_{0}^{t} \eta_{i}(u) e^{-\kappa_{i}^{f}(t-u)} d u, \\
\Phi_{13 i}(t)=\int_{0}^{t} \eta_{i}(u)(t-u) e^{-\kappa_{i}^{f}(t-u)} d u, \quad \Phi_{14 i}(t)=\int_{0}^{t} c(u) V_{i}(u) e^{-\kappa_{i}^{\lambda}(t-u)} d u, \\
\Phi_{15 i}(t)=\int_{0}^{t} c(u) V_{i}(u) e^{-2 \kappa_{i}^{\lambda}(t-u)} d u, \quad \Phi_{16 i}(t)=\int_{0}^{t} c(u) V_{i}(u)(t-u) e^{-\kappa_{i}^{\lambda}(t-u)} d u, \\
\Phi_{17 i}(t)=\int_{0}^{t} c(u) V_{i}(u)(t-u) e^{-2 \kappa_{i}^{\lambda}(t-u)} d u, \quad \Phi_{18 i}(t)=\int_{0}^{t} c(u) V_{i}(u)(t-u)^{2} e^{-2 \kappa_{i}^{\lambda}(t-u)} d u
\end{array}\right.
$$

where $\eta_{i}(t)=V_{i}(t) \sqrt{r(t) c(t)}$. We note in addition that,

$$
\begin{gathered}
\sum_{i=1}^{2 n} \int_{0}^{t} \tilde{\sigma}_{i}^{\lambda}(u, T) d \tilde{W}_{i}(u)=\sum_{i=1}^{n} \rho_{i}^{f \lambda}\left[b_{0 i}+b_{1 i}(T-t)\right] e^{-\kappa_{i}^{\lambda}(T-t)} x_{2 i}(t) \\
+\sum_{i=1}^{n} \sqrt{1-\left(\rho_{i}^{f \lambda}\right)^{2}}\left[b_{0 i}+b_{1 i}(T-t)\right] e^{-\kappa_{i}^{\lambda}(T-t)} x_{3 i}(t)+\sum_{i=1}^{n} \rho_{i}^{f \lambda} b_{1 i} e^{-\kappa_{i}^{\lambda}(T-t)} \Phi_{19 i}(t) \\
\quad+\sum_{i=1}^{n} \sqrt{1-\left(\rho_{i}^{f \lambda}\right)^{2}} b_{1 i} e^{-\kappa_{i}^{\lambda}(T-t)} \Phi_{20 i}(t)
\end{gathered}
$$


where we introduce the state variables

$$
\left\{\begin{array}{l}
x_{2 i}(t)=\int_{0}^{t} \sqrt{c(u) V_{i}(u)} e^{-\kappa_{i}^{\lambda}(t-u)} d \tilde{W}_{i}(u), \quad x_{3 i}(t)=\int_{0}^{t} \sqrt{c(u) V_{i}(u)} e^{-\kappa_{i}^{\lambda}(t-u)} d \tilde{W}_{n+i}(u), \\
\Phi_{19 i}(t)=\int_{0}^{t} c(u) V_{i}(u)(t-u) e^{-\kappa_{i}^{\lambda}(t-u)} d \tilde{W}_{i}(u), \quad \Phi_{20 i}(t)=\int_{0}^{t} c(u) V_{i}(u)(t-u) e^{-\kappa_{i}^{\lambda}(t-u)} d \tilde{W}_{n+i}(u) .
\end{array}\right.
$$

Consequently, the forward credit spread satisfies

$$
\lambda(t, T)=\lambda(0, T)+\sum_{i=1}^{n} \sum_{j=2}^{3} B_{x_{j i}}(T-t) x_{j i}(t)+\sum_{i=1}^{n} \sum_{j=7}^{20} B_{\Phi_{j i}}(T-t) \Phi_{j i}(t),
$$

where the coefficients $B_{x_{j i}}(T-t)$ and $B_{\Phi_{j i}}(T-t)$ are specified in (3.7) and (3.8). It follows from the definition $f^{d}(t, T)=f(t, T)+\lambda(t, T)$ and (A.5) and (A.13) that the defaultable forward rate admits the finite dimensional affine realisation

$$
f^{d}(t, T)=f^{d}(0, T)+\sum_{i=1}^{n} \sum_{j=1}^{3} B_{x_{j i}}(T-t) x_{j i}(t)+\sum_{i=1}^{n} \sum_{j=1}^{20} B_{\Phi_{j i}}(T-t) \Phi_{j i}(t) .
$$

\section{B Proof of Proposition 3.4}

Straightforward application of (A.14) for the affine Markovian forward rate to the definition of the defaultable bond price formula, $\bar{P}^{d}(t, T)=\exp \left(-\int_{t}^{T} f^{d}(t, s) d s\right)$ yields $\bar{P}^{d}(t, T)=\frac{\bar{P}^{d}(0, T)}{\bar{P}^{d}(0, t)} \exp \left(-\sum_{i=1}^{n} \sum_{j=1}^{3} x_{j i}(t) \int_{t}^{T} B_{x_{j i}}(s-t) d s-\sum_{i=1}^{n} \sum_{j=1}^{20} \Phi_{j i}(t) \int_{t}^{T} B_{\Phi_{j i}}(s-t) d s\right)$.

We proceed to integrate the deterministic functions $B_{x_{j i}}$ and $B_{\Phi_{j i}}$ (See Proposition 3.1) in the exponent with respect to maturity. Substituting equations (3.13) - (3.16) into the general exponential defaultable bond price expression (B.1) yields (3.12) in Proposition 3.4 .

\section{References}

Amin, K. and Morton, R. (1995), 'Implied volatility functions in Heath-Jarrow-Morton models', Journal of Financial Economics 35(2), 141-180.

Ball, C. A. and Torous, W. N. (1999), 'The stochastic volatility of short-term interest rates: Some international evidence', Journal of Finance 54(6), 2339-2359.

Berndt, A., Ritchken, P. and Sun, Z. (2010), 'On correlation and default clustering in credit markets', Review of Financial Studies 23(7), 2680-2729. 
Bielecki, T., Jeanblanc, M. and Rutkowski, M. (2007), 'Hedging of basket credit derivatives in credit default swap market', Journal of Credit Risk 3(1), 91-132.

Björk, T., Kabanov, Y. and Runggaldier, W. (1997), 'Bond market structure in the presence of marked point processes', Mathematical Finance 7(2), 211-239.

Björk, T., Landén, C. and Svensson, L. (2004), 'Finite-dimensional Markovian realizations for stochastic volatility forward-rate models', Proceedings of Royal Society London 460,(2041), 53-83.

Brigo, D. and Morini, M. (2005), 'CDS market formulas and models', Working Papers, Banca IMI .

Casassus, J., Collin-Dufresne, P. and Goldstein, R. (2005), 'Unspanned stochastic volatility and fixed income derivatives pricing', Journal of Banking and Finance 29, 2723-2749.

Chan, K. C., Karolyi, G. A., Longstaff, F. A. and Sanders, A. B. (1992), 'An empirical comparison of alternative models of the short-term interest rate', Journal of Finance 47, 1209-1227.

Chiarella, C. and Kwon, O. (2000), 'A complete Markovian stochastic volatility model in the HJM framework', Asia Pacific Financial Markets 7(4), 293-304.

Chiarella, C. and Kwon, O. (2001), 'Classes of interest rate models under the HJM framework', AsiaPacific Financial Markets 8(1), 1-22.

Chiarella, C. and Kwon, O. (2003), 'Finite dimensional affine realisations of HJM models in terms of forward rates and yields', Review of Derivatives Research 6(2), 129-155.

Chiarella, C., Maina, S. C. and Nikitopoulos-Sklibosios, C. (2010), 'Markovian defaultable HJM term structure models with unspanned stochastic volatility', QFRC Working Paper No. 283, Finance Discipline Group, UTS Business School, University of Technology, Sydney .

Chiarella, C., Maina, S. C. and Nikitopoulos-Sklibosios, C. (2011), 'Credit derivative pricing with stochastic volatility models', QFRC Working Paper No. 293, Finance Discipline Group, UTS Business School, University of Technology, Sydney .

Chiarella, C., Nikitopoulos-Sklibosios, C. and Schlögl, E. (2007), 'A Markovian defaultable term structure model with state dependent volatilities', International Journal of Theoretical and Applied Finance 10(1).

D'Souza, D., Amir-Atefi, K. and Racheva-Jotova, B. (2004), Valuation of a credit spread put option: The stable paretian model with copulas, Birkhauser. Handbook of Computational and Numerical Methods in Finance.

Heath, D., Jarrow, R. A. and Morton, A. (1992), 'Bond pricing and the term structure of interest rates: A new methodology for contingent claims valuation', Econometrica 60, 77-105.

Jabbour, G. M., El-Masri, F. and Young, S. D. (2008), 'On the lognormality of forward credit default swap spreads', Journal of Financial Transformation 22, 41-48.

Krekel, M. and Wenzel, J. (2006), 'A unified approach to credit default swaption and constant default swap valuation', Berichte des Fraunhofer ITWM 96.

Li, H. and Zhao, F. (2006), 'Unspanned stochastic volatility: Evidence from hedging interest rate derivatives', Journal of Finance 61, 341-378. 
Mercurio, F. and Moraleda, J. M. (2000), 'An analytically tractable interest rate model with humped volatility', European Journal of Operational Research 120, 205-214.

Pan, J. and Singleton, K. K. (2008), 'Default and recovery implicit in the term structure of sovereign CDS spreads', Journal of Finance 63(5), 2345-2384.

Reno, R. and Uboldi, A. (2005), 'On the presence of unspanned volatility in European interest rate options', Applied Financial Economics Letters 1, 15-18.

Rutkowski, M. and Armstrong, A. (2009), 'Valuation of credit default swaptions and credit default index swaptions', International Journal of Theoretical and Applied Finance 12(7), 1027-1053.

Schönbucher, P. (1998), 'Term structure modelling of defaultable bonds', Review of Derivatives Research 2, 161-192.

Schönbucher, P. (2004), 'A measure of survival', Risk August, 79-85.

Trolle, A. B. and Schwartz, E. S. (2009), 'A general stochastic volatility model for the pricing of interest rate derivatives', The Review of Financial Studies 22(5), 2007-2057. 